\title{
Catalytic Asymmetric Three-Component Acyl-Strecker Reaction
}

\author{
Subhas Chandra Pan and Benjamin List* \\ Max-Planck-Institut für Kohlenforschung, D-45470 Mülheim an der Ruhr, Germany, \\ list@mpi-muelheim.mpg.de
}

\section{Supporting Information}

General: Reactions were monitored by thin layer chromatography using $0.25 \mathrm{~mm}$ E. Merck silica gel coated glass plates (60F-254) using UV light to visualize the course of reaction. Flash column chromatography was performed using E. Merck siliga gel (60, particle size $0.040-0.063 \mathrm{~mm}$ ). Chemical yields refer to pure isolated substances. ${ }^{1} \mathrm{H}$ and ${ }^{13} \mathrm{C}$ NMR spectra were obtained using either a Bruker DPX-300 or AV-400 spectrometer. Chemical shifts are reported in ppm from tetramethylsilane with the solvent resonance as the internal standard. The following abbreviations were used to designate chemical shift mutiplicities: $\mathrm{s}=$ singlet, $\mathrm{d}$ = doublet, $\mathrm{t}=$ triplet, $\mathrm{q}=$ quartet, $\mathrm{h}=$ heptet, $\mathrm{m}=$ multiplet, $\mathrm{br}=$ broad. $\mathrm{MS}(\mathrm{EI})$ : Finnigan MAT 8200 (70 eV), ESI-MS: Finnigan MAT 95, accurate mass determinations: Bruker APEX III FT-MS (7 T magnet).

\section{Preparation of Catalyst}

Catalyst 5 was prepared according to the procedure reported by Jacobsen et al. ${ }^{1}$

\section{General experimental procedure for the catalytic asymmetric three-component acyl-} Strecker reaction

Aldehyde 2 (0.5 mmol), amine 3 (0.5 mmol), and MS 5Å (150 mg) were placed into a dry Schlenk flask. Dry dichloromethane $(2 \mathrm{ml})$ was added and the mixture was stirred for $2 \mathrm{~h}$ at rt. Then catalyst 5 (5 mol\%) was added and the flask was cooled to $-40{ }^{\circ} \mathrm{C}$ and the mixture was stirred for $10 \mathrm{~min}$. Acylcyanide 1 ( $0.75 \mathrm{mmol}$, 1.5 equiv) was added and the mixture was stirred for $36-50 \mathrm{~h}$ at $-40{ }^{\circ} \mathrm{C}$. The mixture was directly subjected to silica gel column chromatography to give pure product 4 .

1. Vachal, P.; Jacobsen, E. N. J. Am. Chem. Soc. 2002, 124, 10012-10014. 
$\overbrace{4 a}^{A C N}$

(4a): Compound 4a was isolated in 94\% yield after silica gel column chromatography (30\% EtOAc in hexane). ${ }^{1} \mathrm{H}$ NMR (400 MHz, $\left.\mathrm{CDCl}_{3}\right) \delta$ 7.44-7.38 (m, 5H), 7.31-7.24 (m, 3H), 7.14-7.07 (m, 3H), 4.58 (d, $J=17.6 \mathrm{~Hz}$, $1 \mathrm{H}), 4.49(\mathrm{~d}, J=12.9 \mathrm{~Hz}, 1 \mathrm{H}), 2.14(\mathrm{~s}, 1 \mathrm{H}) ;{ }^{13} \mathrm{C} \mathrm{NMR}\left(100 \mathrm{MHz}, \mathrm{CDCl}_{3}\right) \delta$ 171.3, 135.3, 131.9, 129.1, 128.8, 128.4, 127.4, 125.9, 116.1, 49.2, 48.3, 21.7; HRMS (EI) calcd for $\mathrm{MH}^{+}$264.126062, found 264.126260; The enantiomeric ratio was determined to be 97.5:2.5 by chiral HPLC (ChiralPak AS-H column, 20\% i-PrOH/heptane, 0.7 mL/min, 220 $\mathrm{nm}, \mathrm{t}_{\mathrm{R}}$ (major $23.3 \mathrm{~min}$ ), $\mathrm{t}_{\mathrm{R}}$ (minor $37.2 \mathrm{~min}$ ).<smiles>COc1ccc(C(C#N)N(C)Cc2ccccc2)cc1</smiles>

(4b): Compound $\mathbf{4 b}$ was isolated in $88 \%$ yield after silica gel column chromatography (30\% EtOAc in hexane). ${ }^{1} \mathrm{H}$ NMR (400 MHz, $\left.\mathrm{CDCl}_{3}\right)$ $\delta$ 7.27-7.13 (m, 5H), 6.97 (d, $J=7.3 \mathrm{~Hz}, 3 \mathrm{H}), 6.80-6.76$ (m, 1H), 4.45 (d, $J=17.6 \mathrm{~Hz}, 1 \mathrm{H}$ ), 4.36 (d, $J=17.4 \mathrm{~Hz}, 2 \mathrm{H}$ ), 3.71 (s, 3H), 2.01 (s, $3 \mathrm{H}) ;{ }^{13} \mathrm{C}$ NMR (100 MHz, $\left.\mathrm{CDCl}_{3}\right) \delta$ 171.2, 160.0, 135.4, 128.9, 128.4, 127.3, 125.9, 123.8, 116.4, 114.2, 55.0, 49.0, 47.7, 21.7; HRMS (EI) calcd for $\mathrm{MH}^{+}$294.137040, found 294.136826; The enantiomeric ratio was determined to be 96.5:3.5 by chiral HPLC (ChiralPak AS-H column, 30\% i-PrOH/heptane, $0.7 \mathrm{~mL} / \mathrm{min}, 220 \mathrm{~nm}$, $\mathrm{t}_{\mathrm{R}}$ (major $24.4 \mathrm{~min}$ ), $\mathrm{t}_{\mathrm{R}}$ (minor $35.6 \mathrm{~min}$ ).

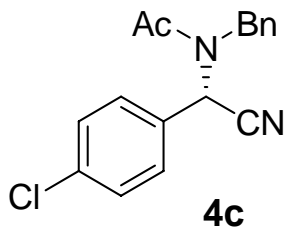

(4c): Compound 4c was isolated in $78 \%$ yield after silica gel column chromatography (25\% EtOAc in hexane). ${ }^{1} \mathrm{H}$ NMR $\left(400 \mathrm{MHz}, \mathrm{CDCl}_{3}\right) \delta$ 7.27-7.14 (m, 7H), 7.00-6.95 (m, 3H), 7.20-7.11 (m, 4H), 7.03 (d, $J=4.1$ $\mathrm{Hz}, 1 \mathrm{H}$ ), 4.47 (d, $J=17.5 \mathrm{~Hz}, 1 \mathrm{H}$ ), 4.40 (d, $J=17.3 \mathrm{~Hz}, 1 \mathrm{H}), 2.06$ (s, 3H); ${ }^{13} \mathrm{C}$ NMR (100 MHz, $\left.\mathrm{CDCl}_{3}\right) \delta 171.2,135.1,134.9,130.6,128.9,128.7,128.5,127.5,125.9$, 115.7, 49.4, 47.8, 21.6 ; HRMS (EI) calcd for $\mathrm{MH}^{+}$298.087328, found 298.087290; The enantiomeric ratio was determined to be 96:4 by chiral HPLC (ChiralPak OJ-H column, 20\% i-PrOH/heptane, $1.0 \mathrm{~mL} / \mathrm{min}, 220 \mathrm{~nm}, \mathrm{t}_{\mathrm{R}}$ (minor $24.9 \mathrm{~min}$ ), $\mathrm{t}_{\mathrm{R}}$ (major $28.8 \mathrm{~min}$ ).

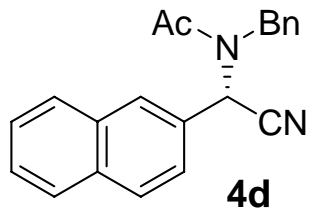

(4d): Compound $\mathbf{4 d}$ was isolated in $92 \%$ yield after silica gel column chromatography (20\% EtOAc in hexane). ${ }^{1} \mathrm{H}$ NMR $\left(400 \mathrm{MHz}, \mathrm{CDCl}_{3}\right) \delta$ 7.88 (s, 1H), 7.75-7.73 (m, 3H), 7.45-7.43 (m, 2H), 7.32 (s, 1H), 7.197.10 (m, 4H), 6.98 (d, $J=7.1 \mathrm{~Hz}, 2 \mathrm{H}), 4.46$ (d, $J=17.6 \mathrm{~Hz}, 1 \mathrm{H}), 4.36$ (d, $J=17.4 \mathrm{~Hz}, 1 \mathrm{H}), 2.05$ (s, 3H); ${ }^{13} \mathrm{C}$ NMR $\left(100 \mathrm{MHz}, \mathrm{CDCl}_{3}\right) \delta 171.4,135.3,133.0,132.5$, 129.2, 127.1, 126.7, 125.9, 124.2, 116.2, 49.2, 48.4, 21.7 ; HRMS (EI) calcd for $\mathrm{MH}^{+}$ 
314.142044, found 314.141915; The enantiomeric ratio was determined to be 97:3 by chiral HPLC (ChiralPak OJ-H column, 30\% i-PrOH/heptane, $1.0 \mathrm{~mL} / \mathrm{min}, 220 \mathrm{~nm}, \mathrm{t}_{\mathrm{R}}$ (minor 29.7 $\min ), t_{R}$ (major $35.4 \mathrm{~min}$ ).

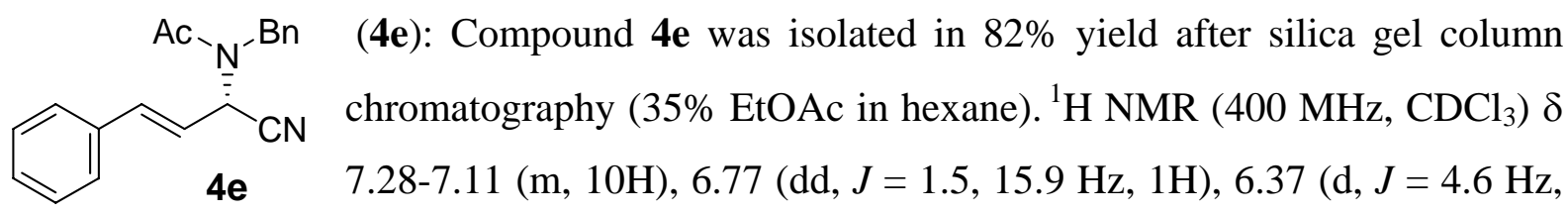
1H), 5.84 (dd, $J=5.4,15.9 \mathrm{~Hz}, 1 \mathrm{H}$ ), 4.62 (d, $J=17.4 \mathrm{~Hz}, 1 \mathrm{H}), 4.49$ (d, $J=17.5 \mathrm{~Hz}, 1 \mathrm{H}) 2.05$ (s, 3H); ${ }^{13} \mathrm{C}$ NMR (100 MHz, $\left.\mathrm{CDCl}_{3}\right) \delta 170.9,135.8,135.6,134.5,128.7,128.5,127.9,127.5$, 126.0, 119.4, 115.7, 49.2, 47.1, 21.5; HRMS (EI) calcd for $\mathrm{MH}^{+}$290.141900, found 290.142060; The enantiomeric ratio was determined to be 96.5:3.5 by chiral HPLC (ChiralPak OJ-H column, 30\% i-PrOH/heptane, $1.0 \mathrm{~mL} / \mathrm{min}, 220 \mathrm{~nm}, \mathrm{t}_{\mathrm{R}}$ (minor $26.3 \mathrm{~min}$ ), $\mathrm{t}_{\mathrm{R}}$ (major $34.2 \mathrm{~min}$ ).

Ac $\widehat{N}^{-B n}$ (4f): Compound $4 \mathbf{f f}$ was isolated in 92\% yield after silica gel column<smiles>CC(C)[CH-]C#N</smiles>
chromatography (50\% EtOAc in hexane). ${ }^{1} \mathrm{H}$ NMR (400 $\left.\mathrm{MHz}, \mathrm{CDCl}_{3}\right) \delta$ 7.32$4 f \quad 7.18(\mathrm{~m}, 5 \mathrm{H}), 5.09(\mathrm{~d}, J=9.7 \mathrm{~Hz}, 1 \mathrm{H}), 4.65(\mathrm{~d}, J=17.4 \mathrm{~Hz}, 1 \mathrm{H}), 4.50$ (d, $J=$ 17.4 Hz, 1H), 2.16-2.05 (m, 1H), 2.00 (s, 3H), 1.05 (d, $J=6.7$ Hz, 3H), 0.86 (d, $J=6.6$ Hz, $3 \mathrm{H}) ;{ }^{13} \mathrm{C} \mathrm{NMR}\left(100 \mathrm{MHz}, \mathrm{CDCl}_{3}\right) \delta 171.2,135.6,128.7,127.6,125.9,117.0,52.6,50.6$, 30.4, 21.8, 18.9, 18.5; HRMS (ESI) calcd for $\mathrm{MNa}^{+}$253.130979, found 253.131132; The enantiomeric ratio was determined to be 96:4 by chiral HPLC (ChiralPak OJ-H column, 3\% i$\mathrm{PrOH} /$ heptane, $1.0 \mathrm{~mL} / \mathrm{min}, 220 \mathrm{~nm}, \mathrm{t}_{\mathrm{R}}$ (minor $36.5 \mathrm{~min}$ ), $\mathrm{t}_{\mathrm{R}}$ (major $38.8 \mathrm{~min}$ ).

Ac ${ }_{N^{-B n}}$ (4g): Compound $4 \mathbf{g}$ was isolated in 46\% yield after silica gel column $>$ CN chromatography (25\% EtOAc in hexane). ${ }^{1} \mathrm{H}$ NMR (400 MHz, $\left.\mathrm{CDCl}_{3}\right) \delta 7.32-$ $4 g \quad 7.14(\mathrm{~m}, 5 \mathrm{H}), 5.68$ (br s, 1H), 4.77 (d, $J=17.7 \mathrm{~Hz}, 1 \mathrm{H}), 4.59$ (d, $J=17.7 \mathrm{~Hz}, 1 \mathrm{H})$, 1.89 (s, 3H), 1.05 (s, 9H); $\left.{ }^{13} \mathrm{C} \mathrm{NMR} \mathrm{(100} \mathrm{MHz,} \mathrm{CDCl}_{3}\right) \delta 172.0,136.3,128.7,127.3,125.3$, 116.7, 55.0, 51.5, 37.4, 26.5, 22.1; HRMS (ESI) calcd for $\mathrm{MNa}^{+}$267.146628, found 267.146781; The enantiomeric ratio was determined to be 97:3 by chiral HPLC (ChiralPak OJ-H column, 5\% i-PrOH/heptane, $1.0 \mathrm{~mL} / \mathrm{min}, 220 \mathrm{~nm}, \mathrm{t}_{\mathrm{R}}$ (major $13.1 \mathrm{~min}$ ), $\mathrm{t}_{\mathrm{R}}$ (minor 16.0 $\min )$.

Ac $N_{N^{-B n}}$ (4h): Compound $4 \mathbf{h}$ was isolated in $75 \%$ yield after silica gel column chromatography (30\% EtOAc in hexane). ${ }^{1} \mathrm{H}$ NMR (400 $\left.\mathrm{MHz}, \mathrm{CDCl}_{3}\right) \delta$ $4 \mathrm{~h}$ 
7.33-7.17 (m, 5H), 5.42 (t, $J=7.0 \mathrm{~Hz}, 1 \mathrm{H}), 4.67$ (d, $J=17.5 \mathrm{~Hz}, 1 \mathrm{H}), 4.52$ (d, $J=17.5 \mathrm{~Hz}$, $1 \mathrm{H}), 2.03$ (s, 3H), 1.71-1.56 (m, 2H), 1.36-1.19 (m, 4H), $0.79(\mathrm{t}, J=7.0 \mathrm{~Hz}, 3 \mathrm{H}) ;{ }^{13} \mathrm{C} \mathrm{NMR}$ $\left(100 \mathrm{MHz}, \mathrm{CDCl}_{3}\right) \delta 170.9,135.8,128.7,127.6,125.8,117.6,49.8,46.1,31.2,27.5,21.6$, 21.5, 13.3; HRMS (ESI) calcd for $\mathrm{MNa}^{+}$267.146579, found 267.146785; The enantiomeric ratio was determined to be 94:6 by chiral HPLC (ChiralPak OJ-H column, 3\% iPrOH/heptane, $0.7 \mathrm{~mL} / \mathrm{min}, 220 \mathrm{~nm}, \mathrm{t}_{\mathrm{R}}$ (minor $42.7 \mathrm{~min}$ ), $\mathrm{t}_{\mathrm{R}}$ (major $45.5 \mathrm{~min}$ ).<smiles>CC1(C)CC(C#N)C(Cc2ccccc2)C1</smiles>

$4 \mathbf{i}$

(4i): Compound $4 \mathbf{i}$ was isolated in $97 \%$ yield after silica gel column chromatography (30\% EtOAc in hexane). ${ }^{1} \mathrm{H}$ NMR (400 MHz, $\left.\mathrm{CDCl}_{3}\right) \delta 7.33$ 7.18 (m, 5H), 5.35 (d, $J=5.8 \mathrm{~Hz}, 1 \mathrm{H}), 4.64$ (d, $J=17.4 \mathrm{~Hz}, 1 \mathrm{H}), 4.51$ (d, $J=$ $17.4 \mathrm{~Hz}, 1 \mathrm{H}$ ), 2.00 (s, 3H), 1.83 (dd, $J=9.6,13.9 \mathrm{~Hz}, 1 \mathrm{H}$ ), 1.43 (dd, $J=4.0,14.0 \mathrm{~Hz}, 1 \mathrm{H}$ ), 0.89 (s, 9H); ${ }^{13} \mathrm{C}$ NMR (100 MHz, $\left.\mathrm{CDCl}_{3}\right) \delta 170.6,135.7,128.7,127.6,126.1,118.8,50.2$, 45.5, 42.8, 29.9, 28.8, 21.7; HRMS (EI) calcd for $\mathrm{MH}^{+}$258.173447, found 258.173211; The enantiomeric ratio was determined to be $96: 4$ by chiral HPLC (ChiralPak OJ-H column, 3\% iPrOH/heptane, $0.7 \mathrm{~mL} / \mathrm{min}, 220 \mathrm{~nm}, \mathrm{t}_{\mathrm{R}}$ (major $28.2 \mathrm{~min}$ ), $\mathrm{t}_{\mathrm{R}}$ (minor $32.9 \mathrm{~min}$ ).

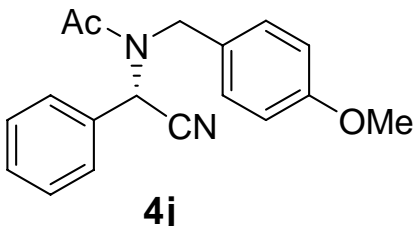

(4j): Compound $\mathbf{4 j}$ was isolated in $95 \%$ yield after silica gel column chromatography (30\% EtOAc in hexane). ${ }^{1} \mathrm{H}$ NMR (400 $\left.\mathrm{MHz}, \mathrm{CDCl}_{3}\right) \delta$ 7.32-7.28 (m, 5H), $6.99(\mathrm{~s}, 1 \mathrm{H}), 6.88$ (d, $J=8.0$ Hz, 2H), 6.73-6.69 (m, 2H), 4.42 (d, $J=17.2 \mathrm{~Hz}, 1 \mathrm{H}), 4.31$ (d, $J=$ $17.0 \mathrm{~Hz}, 1 \mathrm{H}), 3.68$ (s, 3H), 2.04 (s, 3H); $\left.{ }^{13} \mathrm{C} \mathrm{NMR} \mathrm{(100} \mathrm{MHz,} \mathrm{CDCl}_{3}\right) \delta 171.2,158.8,132.0$, 129.0, 128.8, 128.5, 127.3, 127.1, 116.2, 113.8, 54.9, 48.8, 48.2, 21.7; HRMS (ESI) calcd for $\mathrm{MNa}^{+}$317.125793, found 317.126045; The enantiomeric ratio was determined to be $97: 3$ by chiral HPLC (ChiralPak AS-H column, 20\% i-PrOH/heptane, $1.0 \mathrm{~mL} / \mathrm{min}, 220 \mathrm{~nm}, \mathrm{t}_{\mathrm{R}}$ (major $24.6 \mathrm{~min}), \mathrm{t}_{\mathrm{R}}$ (minor $\left.44.9 \mathrm{~min}\right)$.

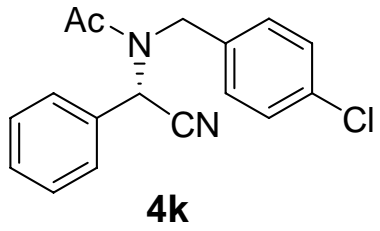

(4k): Compound $\mathbf{4 k}$ was isolated in 93\% yield after silica gel column chromatography (35\% EtOAc in hexane). ${ }^{1} \mathrm{H}$ NMR (400 $\left.\mathrm{MHz}, \mathrm{CDCl}_{3}\right) \delta$ 7.41-7.36 (m, 5H), 7.24 (d, $\left.J=8.4 \mathrm{~Hz}, 2 \mathrm{H}\right), 7.14$ (s, 1H), 6.98 (d, $J=8.1 \mathrm{~Hz}, 2 \mathrm{H}), 4.55$ (d, $J=17.6 \mathrm{~Hz}, 1 \mathrm{H}), 4.45$ (d, $J=$ 17.4 Hz, 1H), 2.12 (s, 3H); ${ }^{13} \mathrm{C}$ NMR (100 MHz, $\left.\mathrm{CDCl}_{3}\right) \delta 171.0,133.8,133.2,131.7,129.2$, 129.0, 128.6, 127.3, 116.0, 48.6, 48.2, 21.6; HRMS (ESI) calcd for $\mathrm{MNa}^{+}$321.076391, found 321.076513; The enantiomeric ratio was determined to be 97.5:2.5 by chiral HPLC 
(ChiralPak AS-H column, 20\% i-PrOH/heptane, $0.7 \mathrm{~mL} / \mathrm{min}, 220 \mathrm{~nm}, \mathrm{t}_{\mathrm{R}}$ (major $23.3 \mathrm{~min}$ ), $\mathrm{t}_{\mathrm{R}}$ (minor $39.3 \mathrm{~min}$ ).<smiles>N#CC(c1ccccc1)N(Cc1ccccc1)Cc1ccccc1</smiles>

4

(4l): Compound $\mathbf{4 l}$ was isolated in 92\% yield after silica gel column chromatography (30\% EtOAc in hexane). ${ }^{1} \mathrm{H} \mathrm{NMR}\left(400 \mathrm{MHz}, \mathrm{CDCl}_{3}\right.$ ) $\delta$ 7.90-7.87 (m, 1H), 7.78 (d, $J=8.2 \mathrm{~Hz}, 2 \mathrm{H}), 7.56-7.51$ (m, 2H), 7.47 (br s, 2H), 7.42 (t, $J=7.5 \mathrm{~Hz}, 1 \mathrm{H}), 7.36-7.34$ (m, 3H), 7.28-7.23 (m, 2H), 5.10 (d, $J=18.2 \mathrm{~Hz}, 1 \mathrm{H}), 4.98$ (d, $J=18.0 \mathrm{~Hz}, 1 \mathrm{H}), 2.16$ (s, 3H);

${ }^{13} \mathrm{C}$ NMR $\left(100 \mathrm{MHz}, \mathrm{CDCl}_{3}\right) \delta$ 171.6, 133.3, 131.9, 129.8, 129.0, 128.8, 128.7, 128.0, 127.3, 126.2, 125.7, 124.9, 122.8, 121.4, 116.0, 48.4, 46.9, 21.2; HRMS (ESI) calcd for $\mathrm{MNa}^{+}$ 337.131117, found 337.131132; The enantiomeric ratio was determined to be $97: 3$ by chiral HPLC (ChiralPak AS-H column, 20\% i-PrOH/heptane, $0.7 \mathrm{~mL} / \mathrm{min}, 220 \mathrm{~nm}$, $\mathrm{t}_{\mathrm{R}}$ (major 35.5 $\min ), t_{R}(\operatorname{minor} 42.9 \min )$.

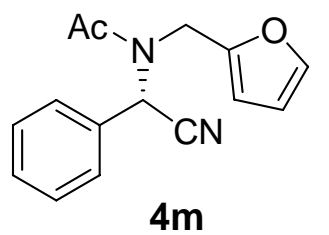

(4m): Compound $\mathbf{4 m}$ was isolated in $83 \%$ yield after silica gel column chromatography (40\% EtOAc in hexane). ${ }^{1} \mathrm{H}$ NMR (400 $\left.\mathrm{MHz}, \mathrm{CDCl}_{3}\right) \delta$ 7.32-7.27 (m, 5H), 7.21 (t, $J=0.7 \mathrm{~Hz}, 1 \mathrm{H}), 6.93$ (s, 1H), 6.13 (d, $J=2.5$ Hz, 1H), 5.87 (s, 1H), 4.40 (d, $J=17.2 \mathrm{~Hz}, 1 \mathrm{H}), 4.22$ (d, $J=17.1 \mathrm{~Hz}$, 1H), 2.26 (s, 3H); ${ }^{13} \mathrm{C}$ NMR (100 MHz, $\left.\mathrm{CDCl}_{3}\right) \delta 170.9,142.2,131.5,130.2,128.9,128.7$, 127.9, 127.1, 110.2, 108.8, 47.5, 42.1, 21.3; HRMS (ESI) calcd for $\mathrm{MNa}^{+}$277.095302, found 277.095314; The enantiomeric ratio was determined to be 90:10 by chiral HPLC (ChiralPak AS-H column, 20\% i-PrOH/heptane, $1.0 \mathrm{~mL} / \mathrm{min}, 220 \mathrm{~nm}$, $\mathrm{t}_{\mathrm{R}}$ (major $16.4 \mathrm{~min}$ ), $\mathrm{t}_{\mathrm{R}}$ (minor 31.0 $\min )$.

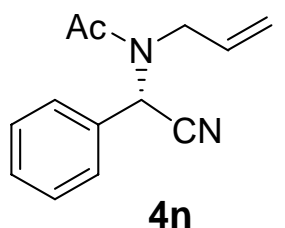

(4n): Compound 4n was isolated in $88 \%$ yield after silica gel column chromatography (30\% EtOAc in hexane). ${ }^{1} \mathrm{H}$ NMR (400 $\left.\mathrm{MHz}, \mathrm{CDCl}_{3}\right) \delta$ 7.39-7.32 (m, 5H), 6.98 (s, 1H), 5.57-7.47 (m, 1H), 5.05 (dd, $J=11.4 \mathrm{~Hz}$, 13.3 Hz, 2H), 3.87-3.73 (m, 2H), 2.11 (s, 3H); ${ }^{13} \mathrm{C}$ NMR (100 MHz, $\left.\mathrm{CDCl}_{3}\right) \delta 170.8,132.0,131.9,129.0,128.8,127.2,117.7,116.3,48.0,47.6,21.2$; HRMS (ESI) calcd for $\mathrm{MNa}^{+}$237.099802, found 237.099833; The enantiomeric ratio was determined to be $94: 6$ by chiral HPLC (ChiralPak AS-H column, 20\% i-PrOH/heptane, $1.0 \mathrm{~mL} / \mathrm{min}, 220$ $\mathrm{nm}, \mathrm{t}_{\mathrm{R}}$ (major $\left.11.3 \mathrm{~min}\right)$, $\mathrm{t}_{\mathrm{R}}$ (minor $22.9 \mathrm{~min}$ ). 


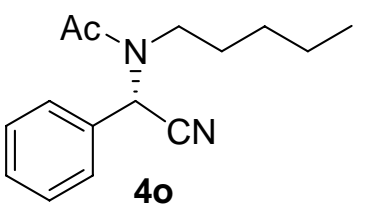

(40): Compound 40 was isolated in 76\% yield after silica gel column chromatography (25\% EtOAc in hexane). ${ }^{1} \mathrm{H}$ NMR (400 MHz, $\mathrm{CDCl}_{3}$ ) 反 7.38-7.32 (m, 5H), 6.95 (s, $1 \mathrm{H}), 3.22-3.14(\mathrm{~m}, 1 \mathrm{H}), 3.11-3.03(\mathrm{~m}$, $1 \mathrm{H}), 2.15$ (s, 3H), 1.58-1.54 (m, 1H), 1.18-1.03 (m, 5H), 0.74 (t, $J=7.0 \mathrm{~Hz}, 3 \mathrm{H}) ;{ }^{13} \mathrm{C}$ NMR $\left(100 \mathrm{MHz}, \mathrm{CDCl}_{3}\right) \delta 170.3,132.3,128.9,128.7,127.0,116.6,47.3,46.0,28.6,28.5,21.6$, 20.8, 13.4 ; HRMS (ESI) calcd for $\mathrm{MNa}^{+}$357.193652, found 357.193728; The enantiomeric ratio was determined to be 87:13 by chiral HPLC (ChiralPak AS-H column, 20\% i$\mathrm{PrOH} / \mathrm{heptane,} 1.0 \mathrm{~mL} / \mathrm{min}, 220 \mathrm{~nm}, \mathrm{t}_{\mathrm{R}}$ (major $8.3 \mathrm{~min}$ ), $\mathrm{t}_{\mathrm{R}}$ (minor $15.3 \mathrm{~min}$ ).<smiles>CCCCCCC(=O)NC(C#N)c1ccccc1</smiles>

$4 p$

(4p): Compound $\mathbf{4 p}$ was isolated in $84 \%$ yield after silica gel column chromatography (10\% EtOAc in hexane). ${ }^{1} \mathrm{H}$ NMR (400 $\left.\mathrm{MHz}, \mathrm{CDCl}_{3}\right) \delta$ 7.34-7.31 (m, 2H), 7.29-7.26 (m, 3H), 7.21-7.13 (m, 3H), 7.06 (brs, 1H), 6.97 (d, $J=6.9 \mathrm{~Hz}, 2 \mathrm{H}), 4.49$ (d, $J=17.4$ Hz, 1H), 4.38 (d, $J=17.4 \mathrm{~Hz}, 1 \mathrm{H}$ ), 2.22 (t, $J=7.2 \mathrm{~Hz}, 2 \mathrm{H}$ ), 1.58-1.53 (m, 2H), 1.21-1.11 (m, $6 \mathrm{H}), 0.78(\mathrm{t}, J=6.8 \mathrm{~Hz}, 3 \mathrm{H}) ;{ }^{13} \mathrm{C}$ NMR $\left(100 \mathrm{MHz}, \mathrm{CDCl}_{3}\right) \delta 173.8,135.5,132.1,128.9$, 128.8, 128.4, 127.3, 125.9, 116.2, 48.6, 48.4, 33.2, 31.1, 28.5, 24.6, 22.0, 13.6; The enantiomeric ratio was determined to be 94:6 by chiral HPLC (ChiralPak AS-H column, 10\% i-PrOH/heptane, $0.7 \mathrm{~mL} / \mathrm{min}, 220 \mathrm{~nm}, \mathrm{t}_{\mathrm{R}}$ (major $14.1 \mathrm{~min}$ ), $\mathrm{t}_{\mathrm{R}}$ (minor $20.1 \mathrm{~min}$ ).
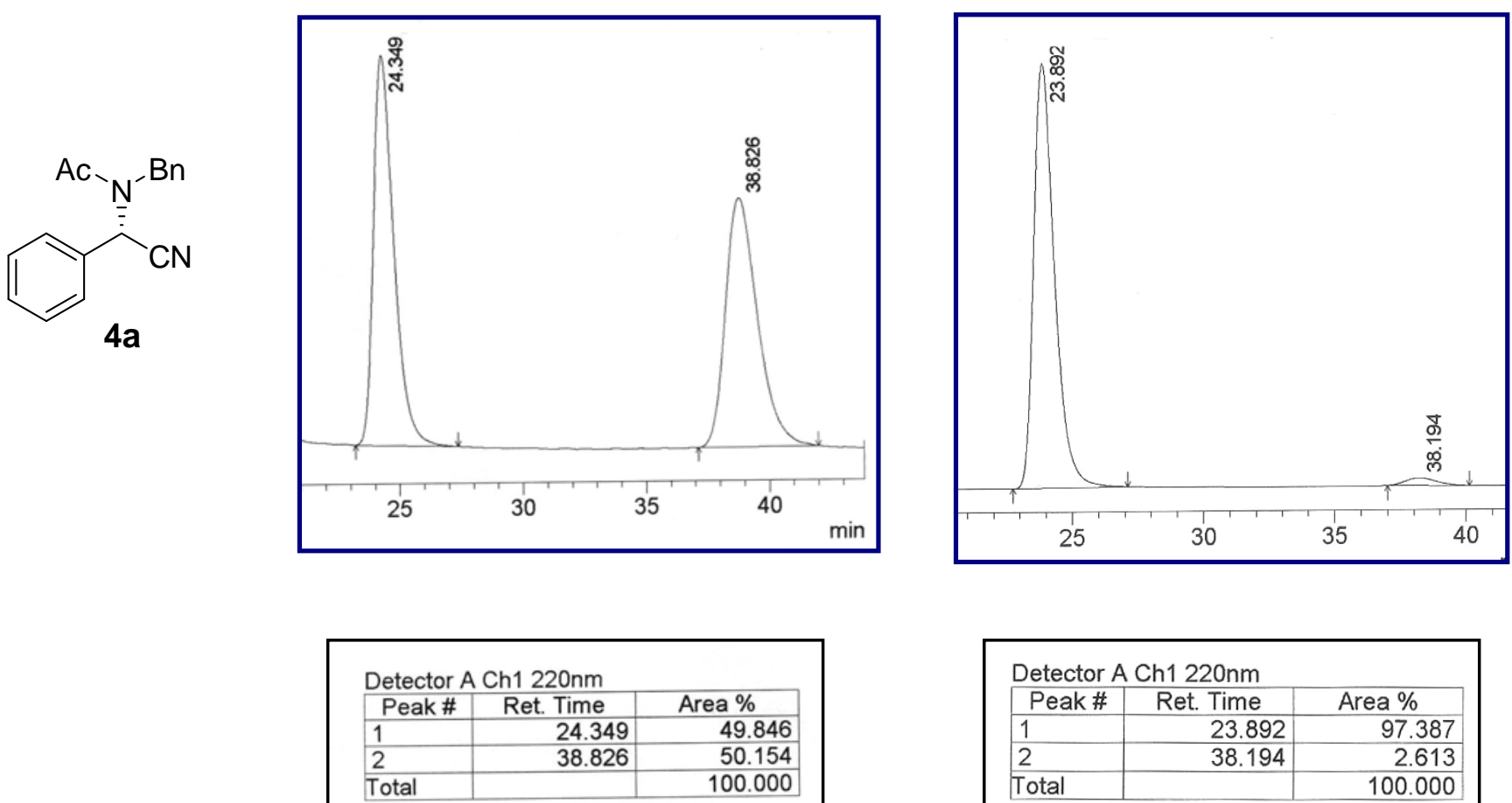

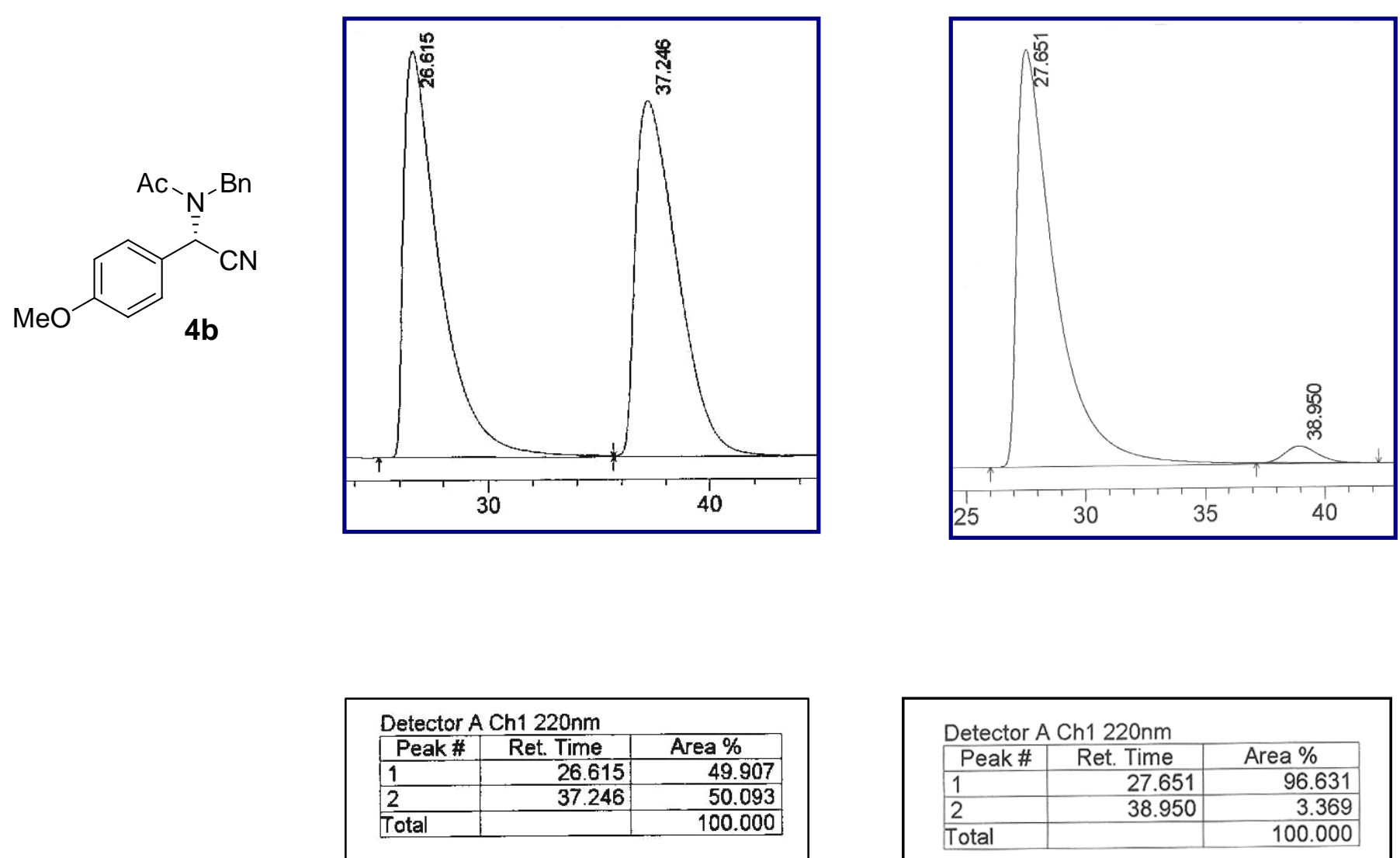

Detector A Ch1 220nm
\begin{tabular}{|l|r|r|}
\hline Peak \# & Ret. Time & Area $\%$ \\
\hline 1 & 27.651 & 96.631 \\
\hline 2 & 38.950 & 3.369 \\
\hline Total & & 100.000 \\
\hline
\end{tabular}



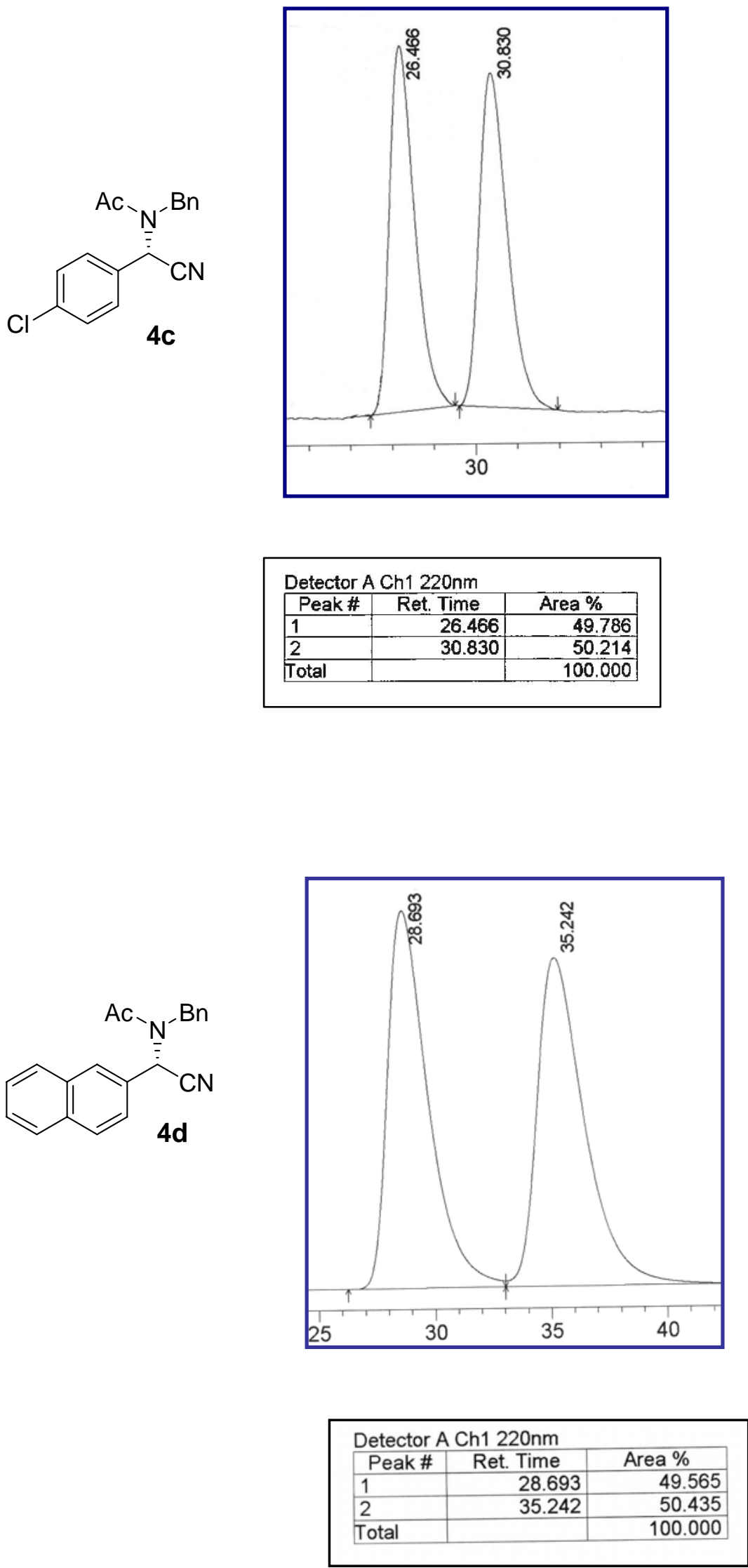
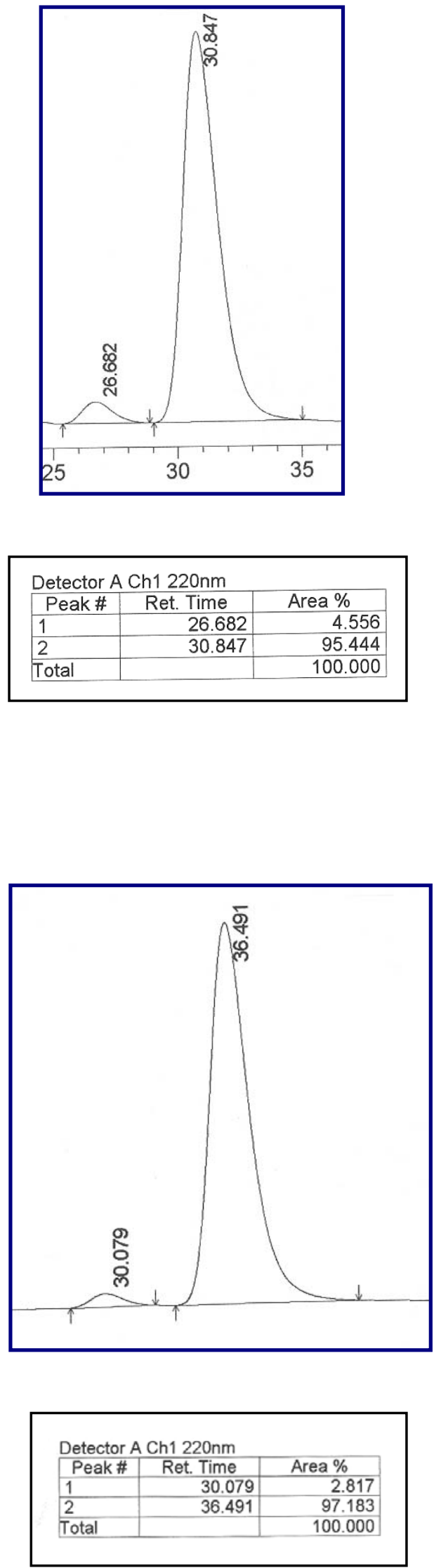

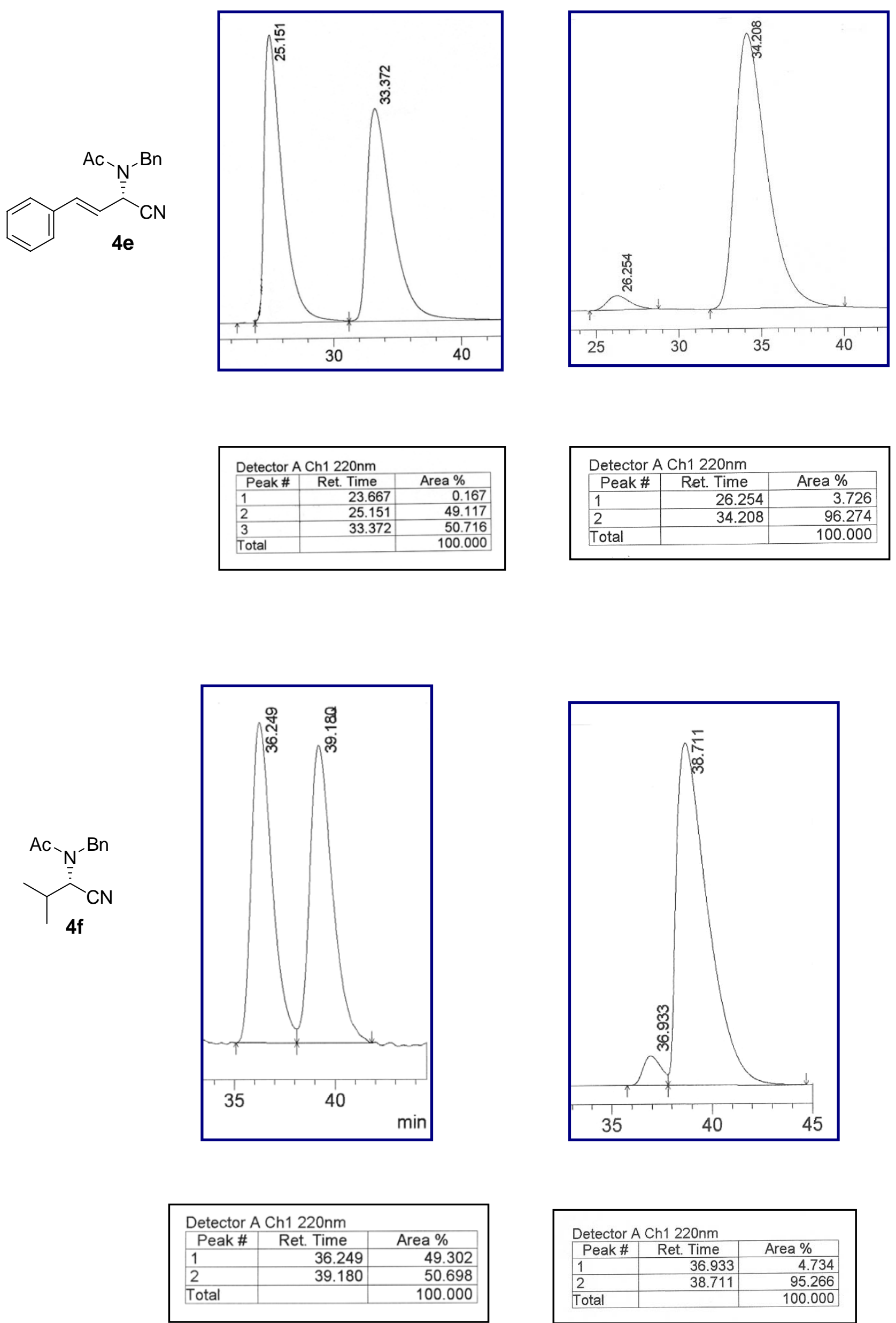


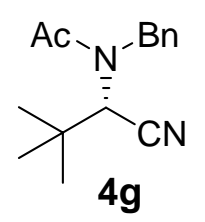

$4 g$
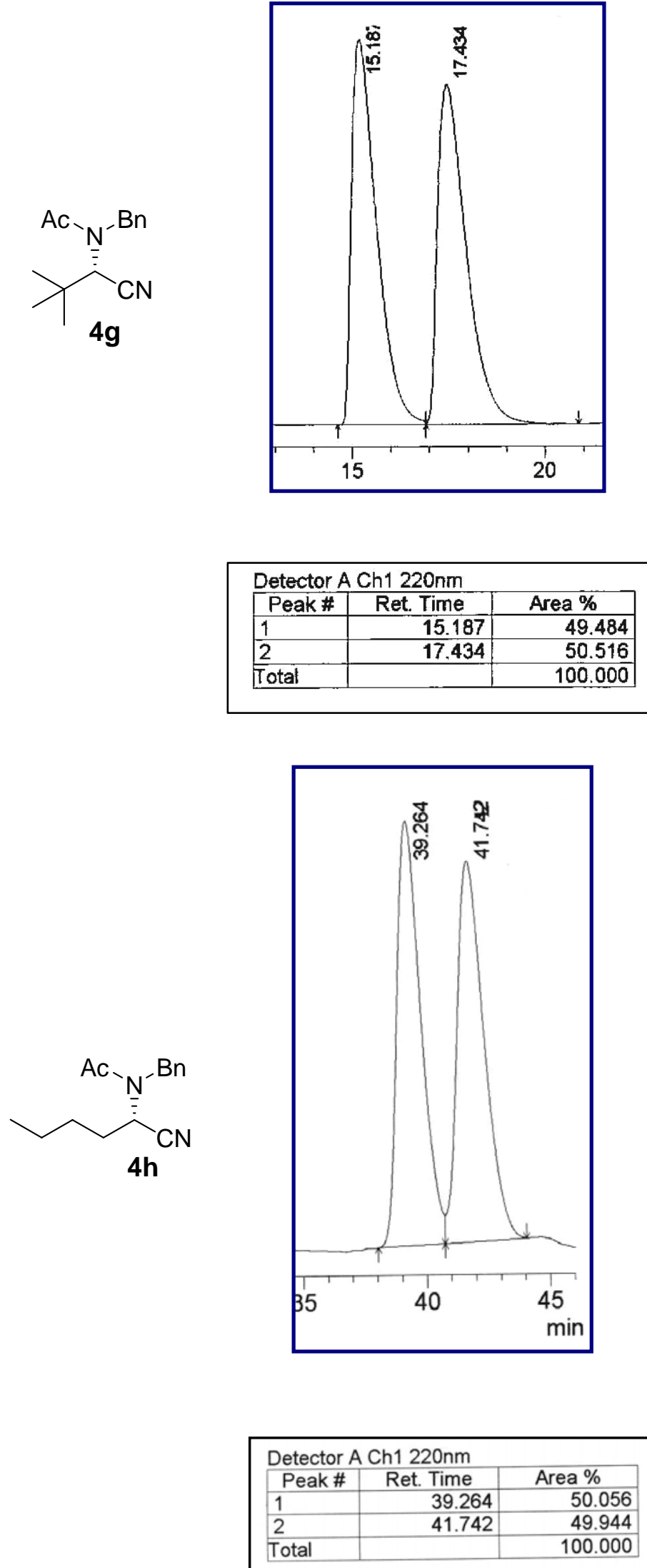
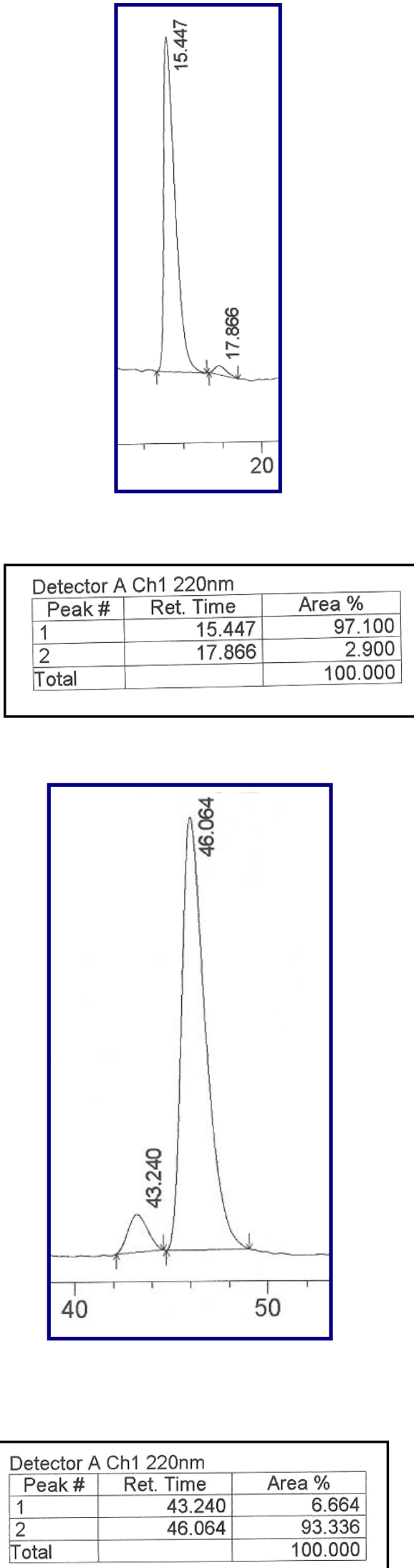
<smiles>CN(Cc1ccccc1)[C@H](C#N)CC(C)(C)C</smiles>

$4 i$

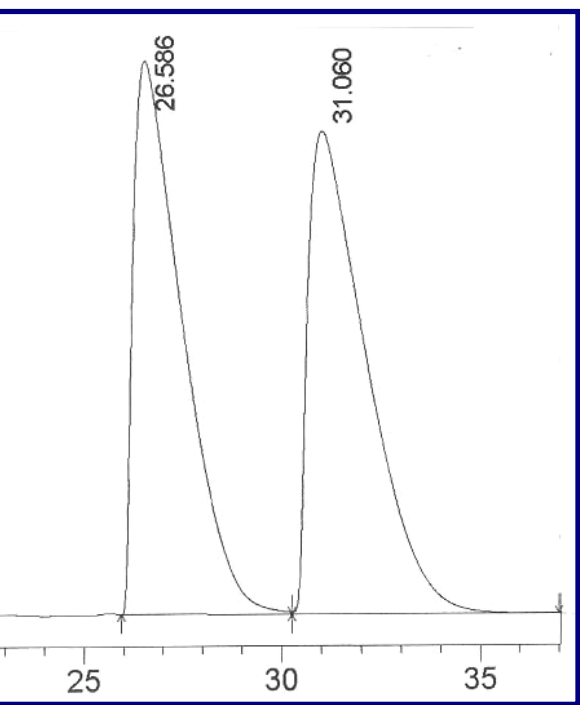

Detector A Ch1 220nm

\begin{tabular}{|l|r|r|}
\hline Peak \# & Ret. Time & \multicolumn{1}{|c|}{ Area \% } \\
\hline 1 & 26.586 & 49.192 \\
\hline 2 & 31.060 & 50.808 \\
\hline Total & & 100.000 \\
\hline
\end{tabular}

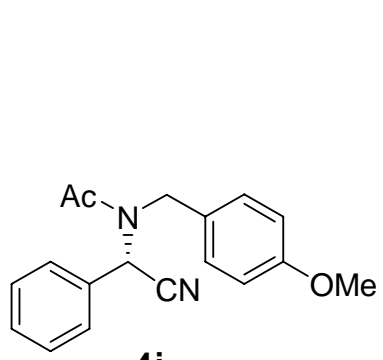

4j

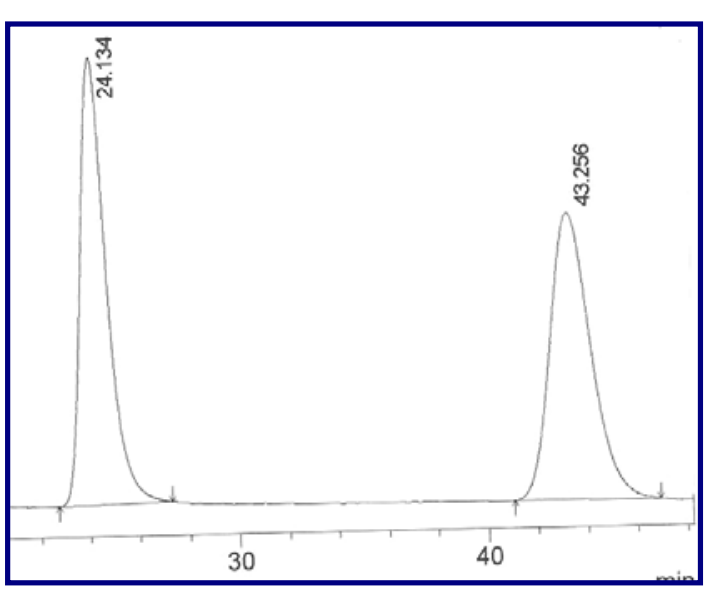

Detector A Ch1 220nm
\begin{tabular}{|l|r|r|}
\hline Peak \# & Ret. Time & Area $\%$ \\
\hline 1 & 24.134 & 49.943 \\
\hline 2 & 43.256 & 50.057 \\
\hline Total & & 100.000 \\
\hline
\end{tabular}

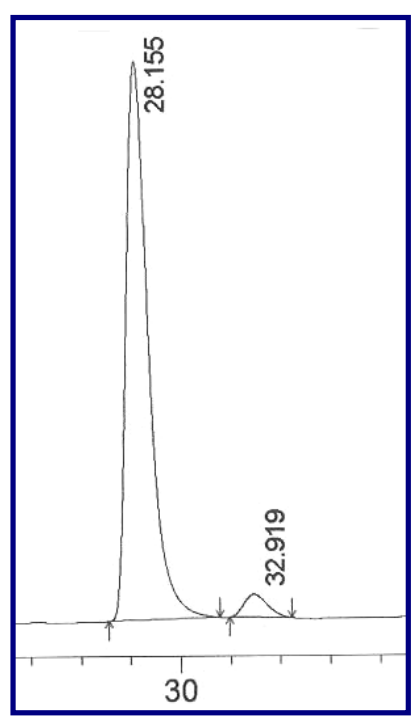

Detector A Ch1 220nm
\begin{tabular}{|l|r|r|}
\hline Peak \# & Ret. Time & Area \% \\
\hline 1 & 28.155 & 95.830 \\
\hline 2 & 32.919 & 4.170 \\
\hline Total & & 100.000 \\
\hline
\end{tabular}

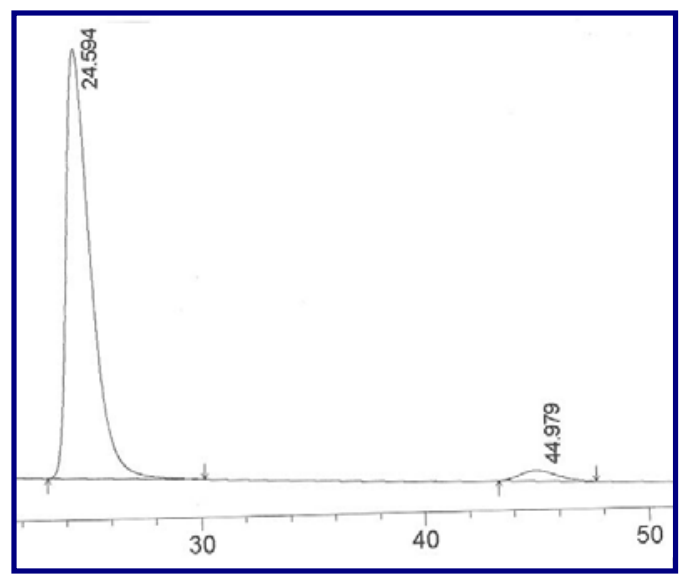

Detector A Ch1 220nm

\begin{tabular}{|l|r|r|}
\hline Peak \# & Ret. Time & \multicolumn{1}{|c|}{ Area \% } \\
\hline 1 & 24.594 & 96.390 \\
\hline 2 & 44.979 & 3.610 \\
\hline Total & & 100.000 \\
\hline
\end{tabular}



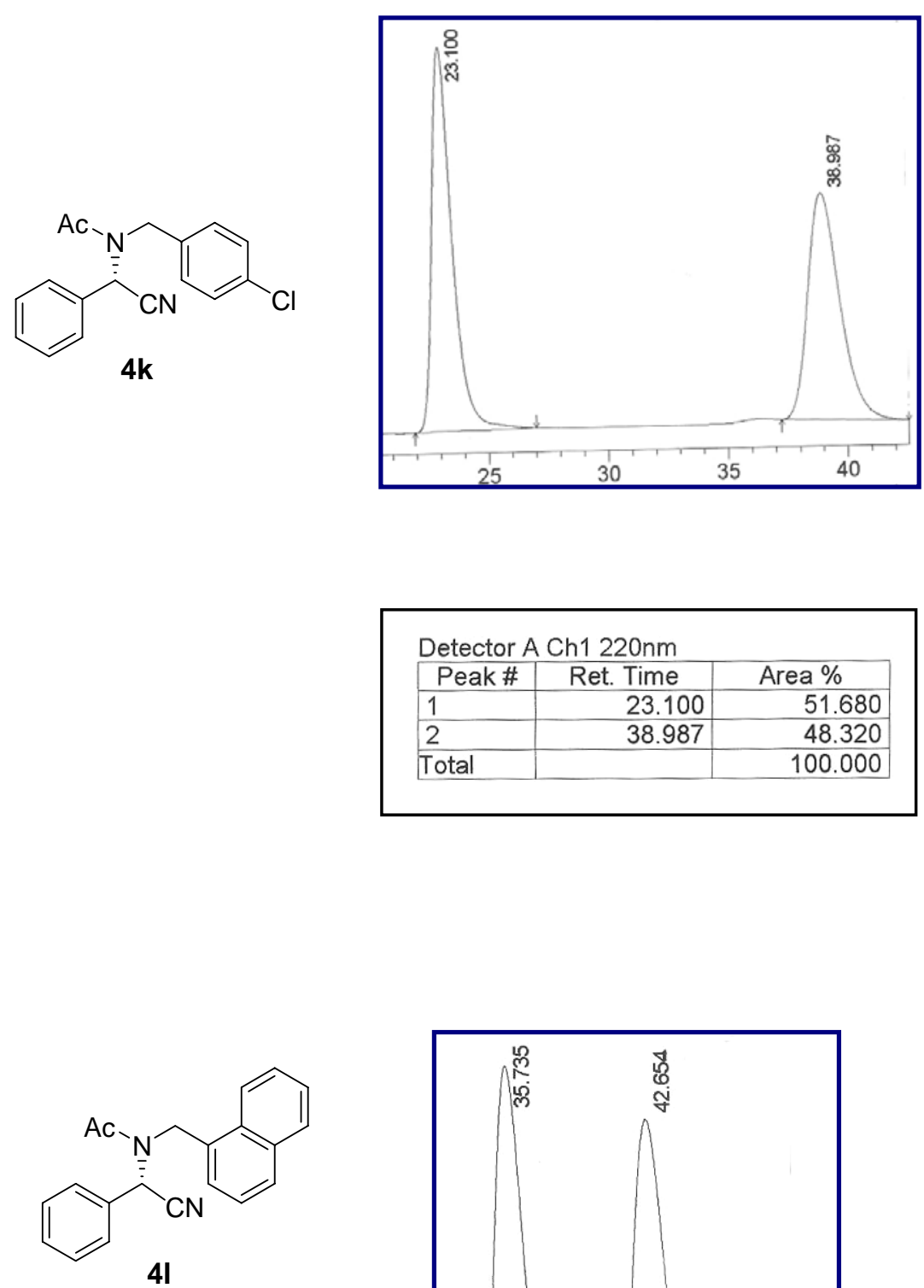

4

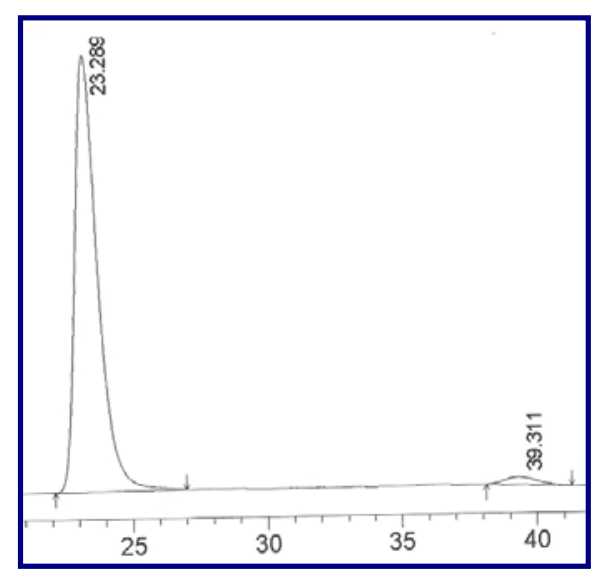

Detector A Ch1 220nm
\begin{tabular}{|l|r|r|}
\hline Peak \# & Ret. Time & Area $\%$ \\
\hline 1 & 23.289 & 97.433 \\
\hline 2 & 39.311 & 2.567 \\
\hline Total & & 100.000 \\
\hline
\end{tabular}

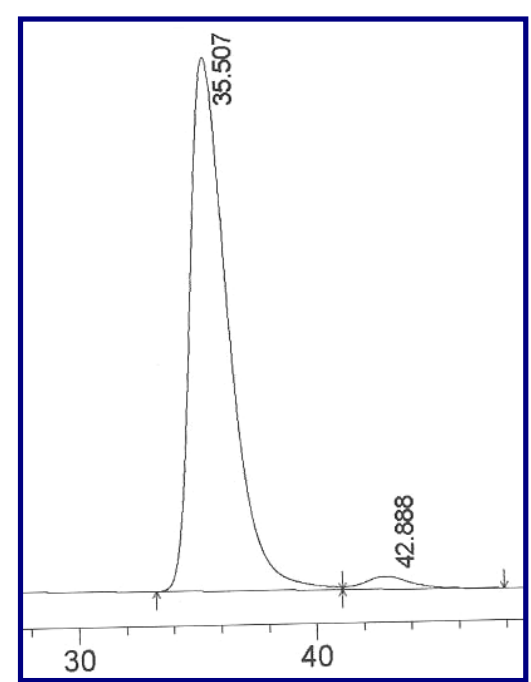

Detector A Ch1 220nm

\begin{tabular}{|l|r|r|}
\hline Peak \# & Ret. Time & \multicolumn{1}{|c|}{ Area \% } \\
\hline 1 & 35.735 & 49.937 \\
\hline 2 & 42.654 & 50.063 \\
\hline Total & & 100.000 \\
\hline
\end{tabular}

Detector A Ch1 220nm
\begin{tabular}{|l|r|r|}
\hline Peak \# & Ret. Time & Area $\%$ \\
\hline 1 & 35.507 & 97.068 \\
\hline 2 & 42.888 & 2.932 \\
\hline Total & & 100.000 \\
\hline
\end{tabular}




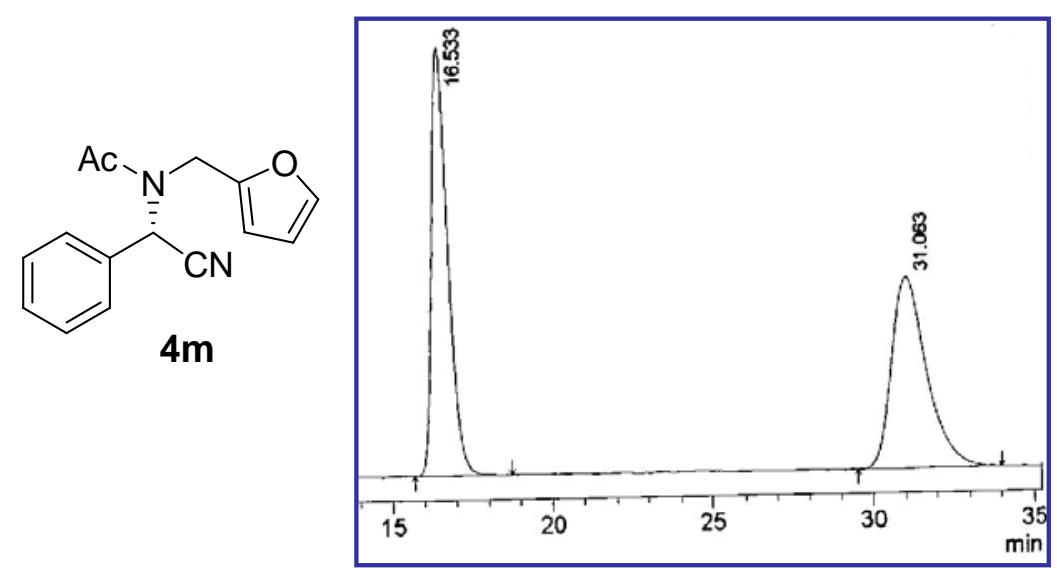

\begin{tabular}{|c|c|c|}
\hline \multicolumn{3}{|c|}{ Detector A Ch1 220nm } \\
\hline Peak \# & Ret. Time & Area \% \\
\hline 1 & 16.533 & 50.480 \\
\hline 2 & 31.063 & 49.520 \\
\hline Total & & 100.000 \\
\hline
\end{tabular}

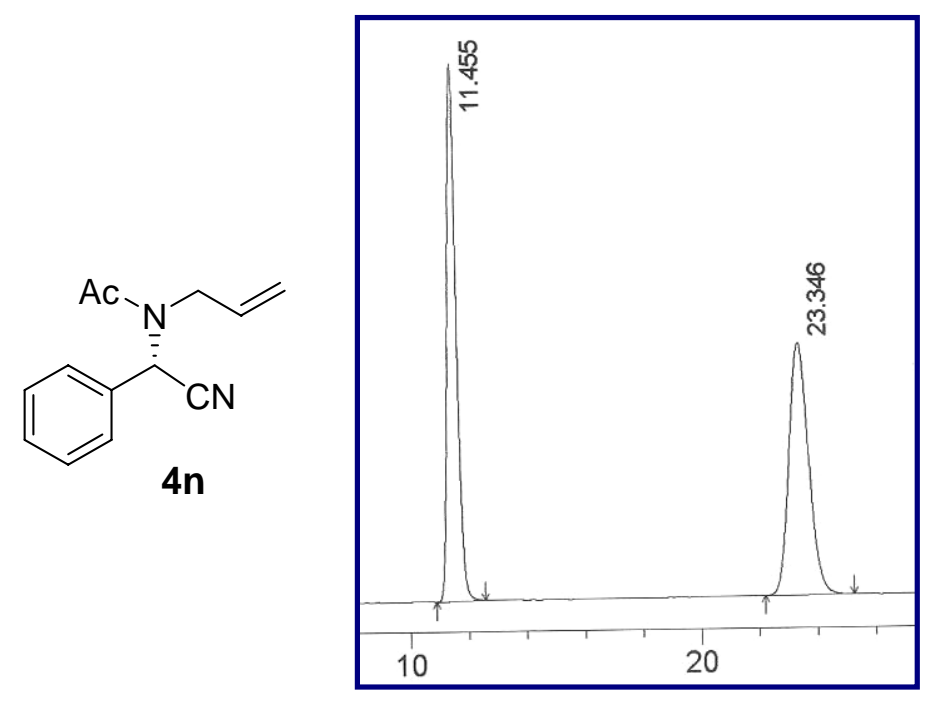

\begin{tabular}{|c|c|c|}
\hline \multicolumn{3}{|c|}{ Detector A Ch1 220nm } \\
\hline Peak \# & Ret. Time & Area \% \\
\hline 1 & 11.455 & 49.934 \\
\hline 2 & 23.346 & 50.066 \\
\hline Total & & 100.000 \\
\hline
\end{tabular}
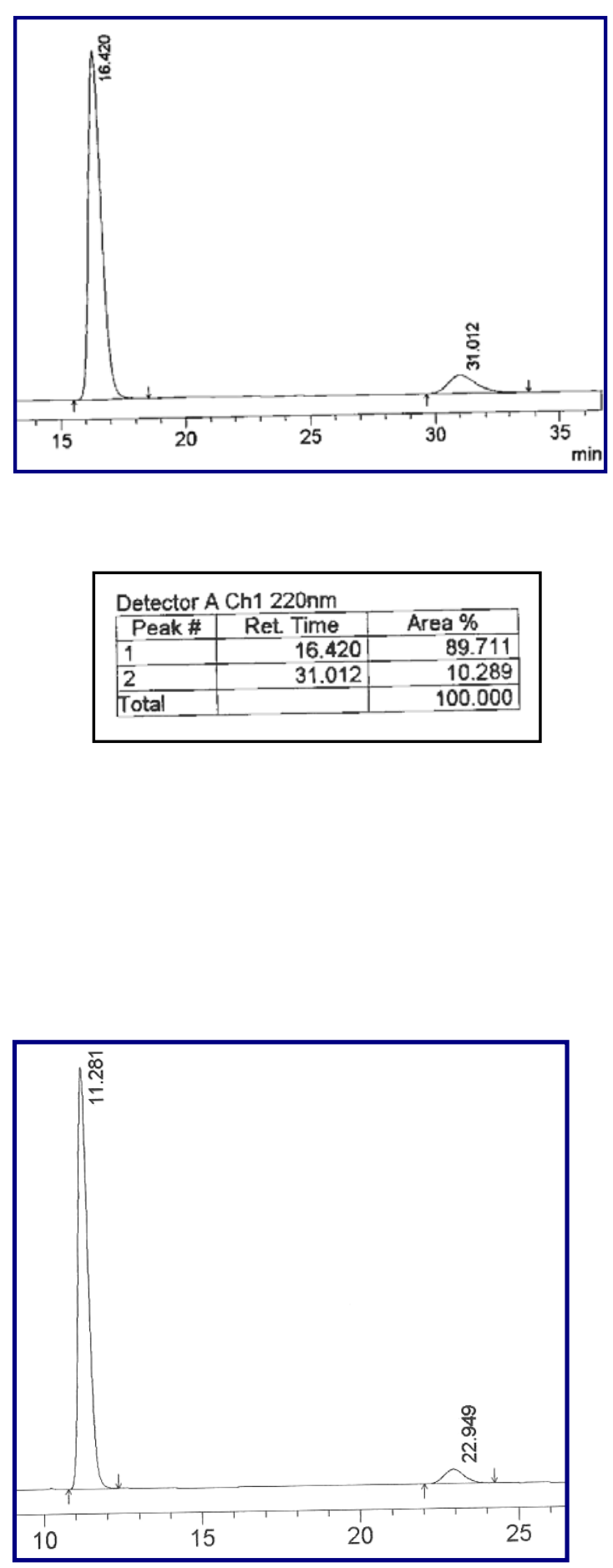

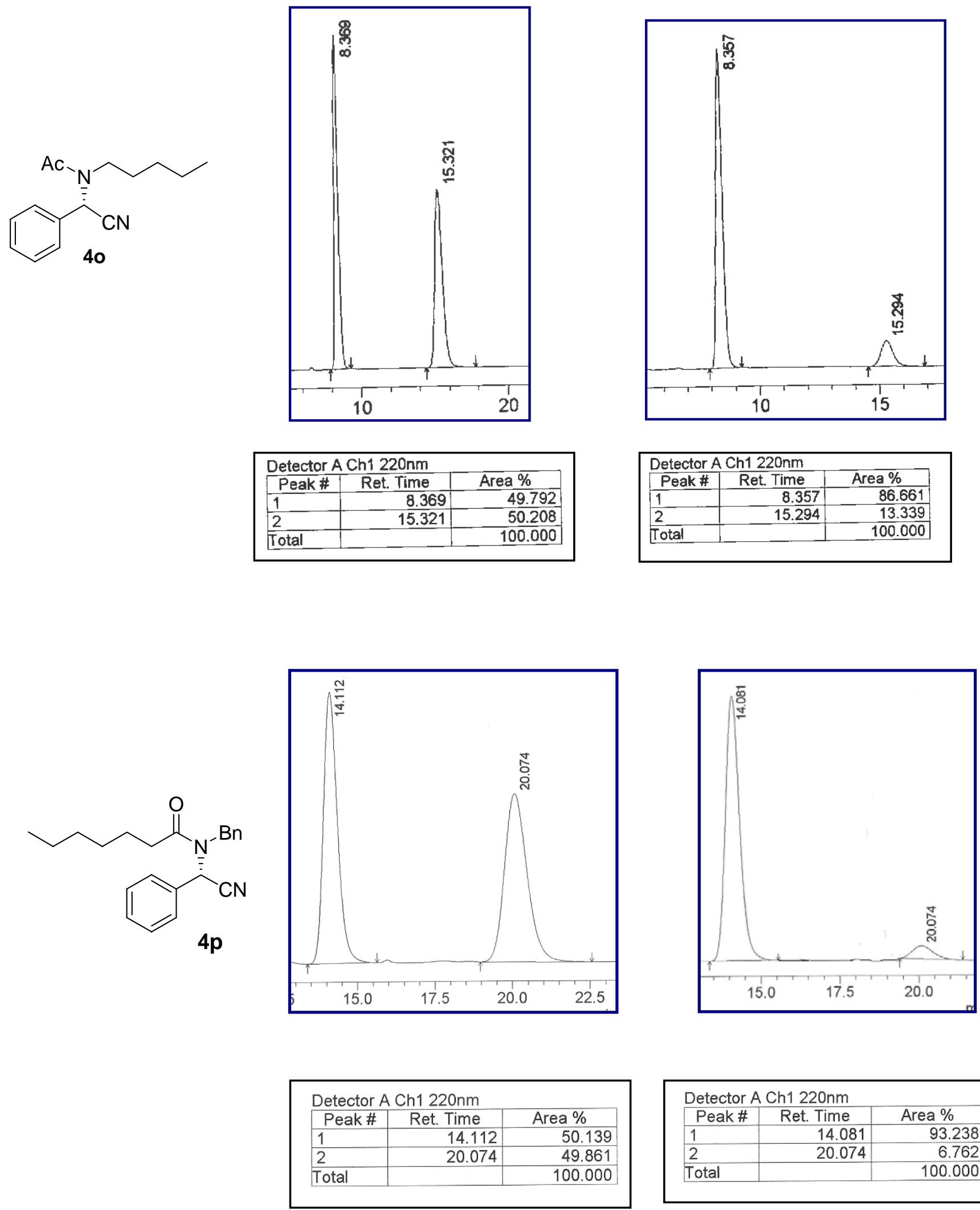

Detector A Ch1 220nm
\begin{tabular}{|l|r|r|}
\hline Peak \# & Ret. Time & Area $\%$ \\
\hline 1 & 14.081 & 93.238 \\
\hline 2 & 20.074 & 6.762 \\
\hline Total & & 100.000 \\
\hline
\end{tabular}



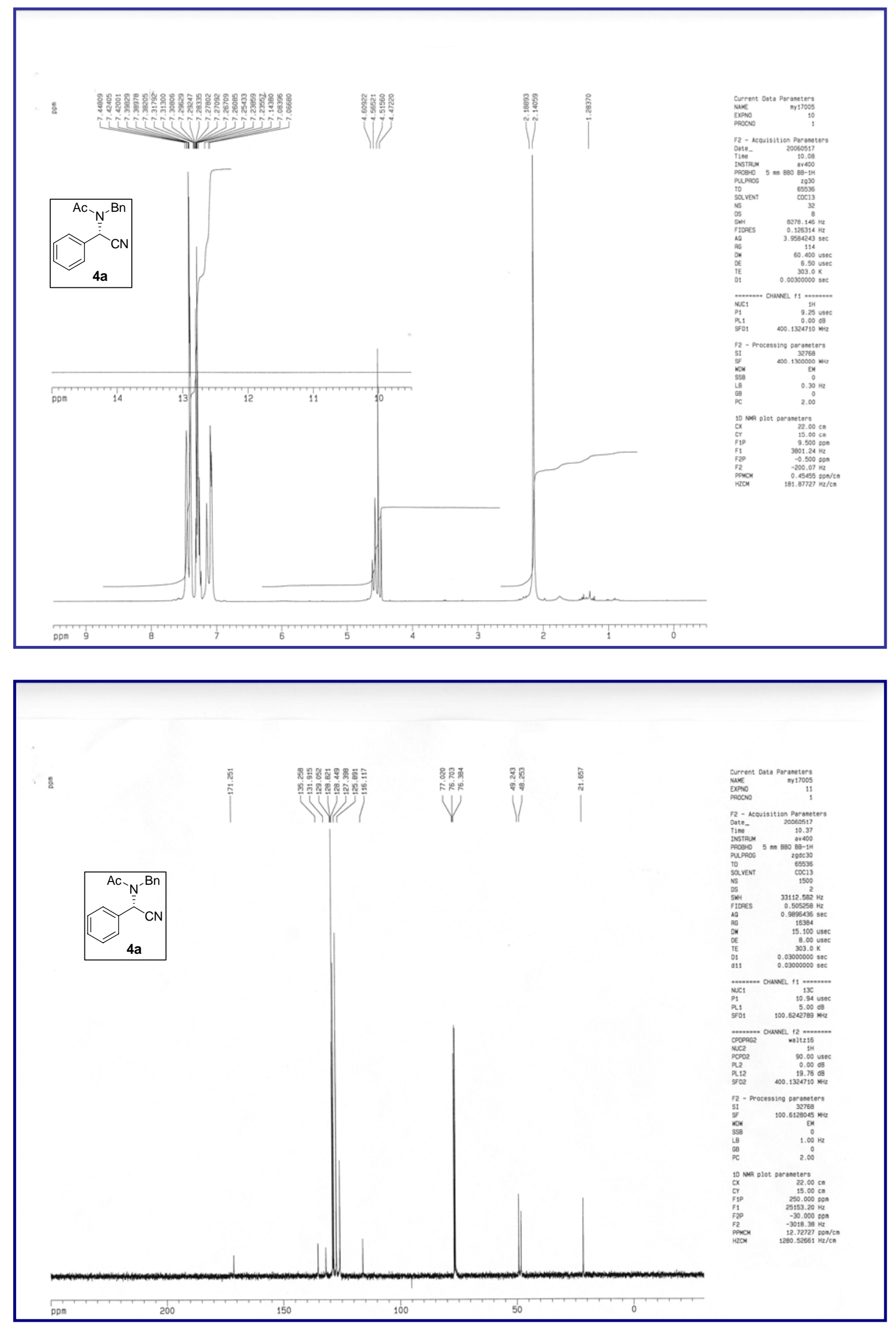

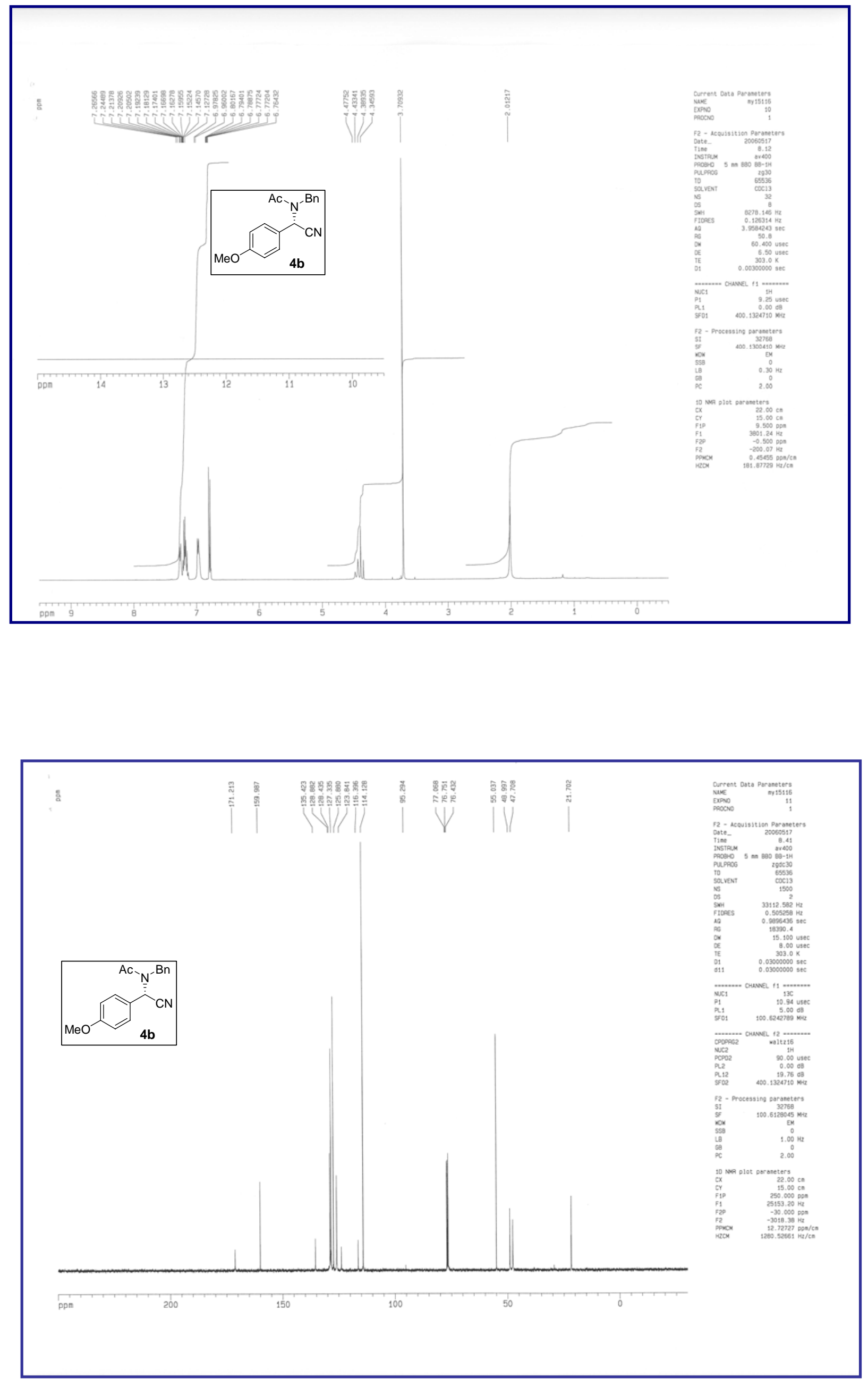

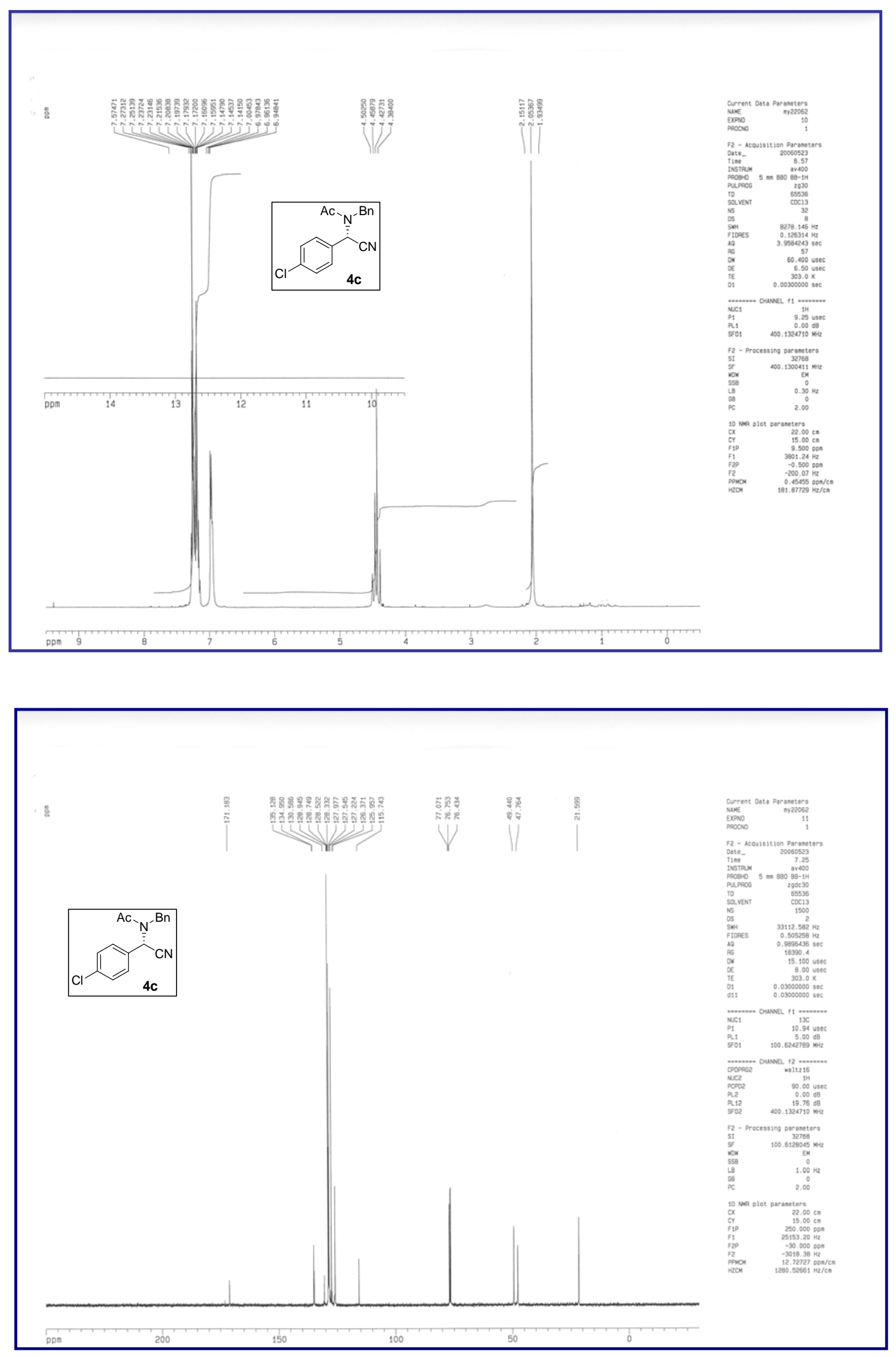

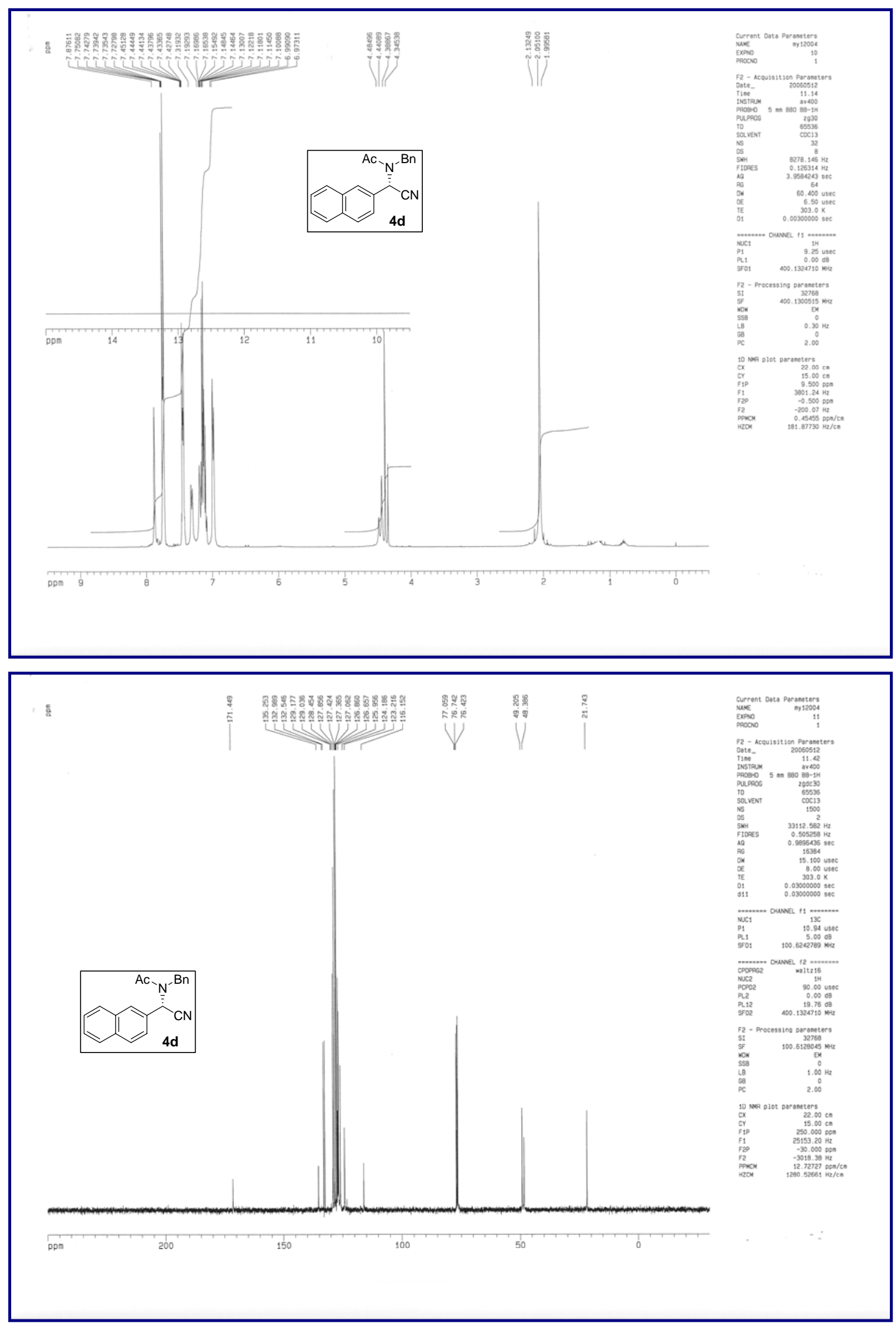

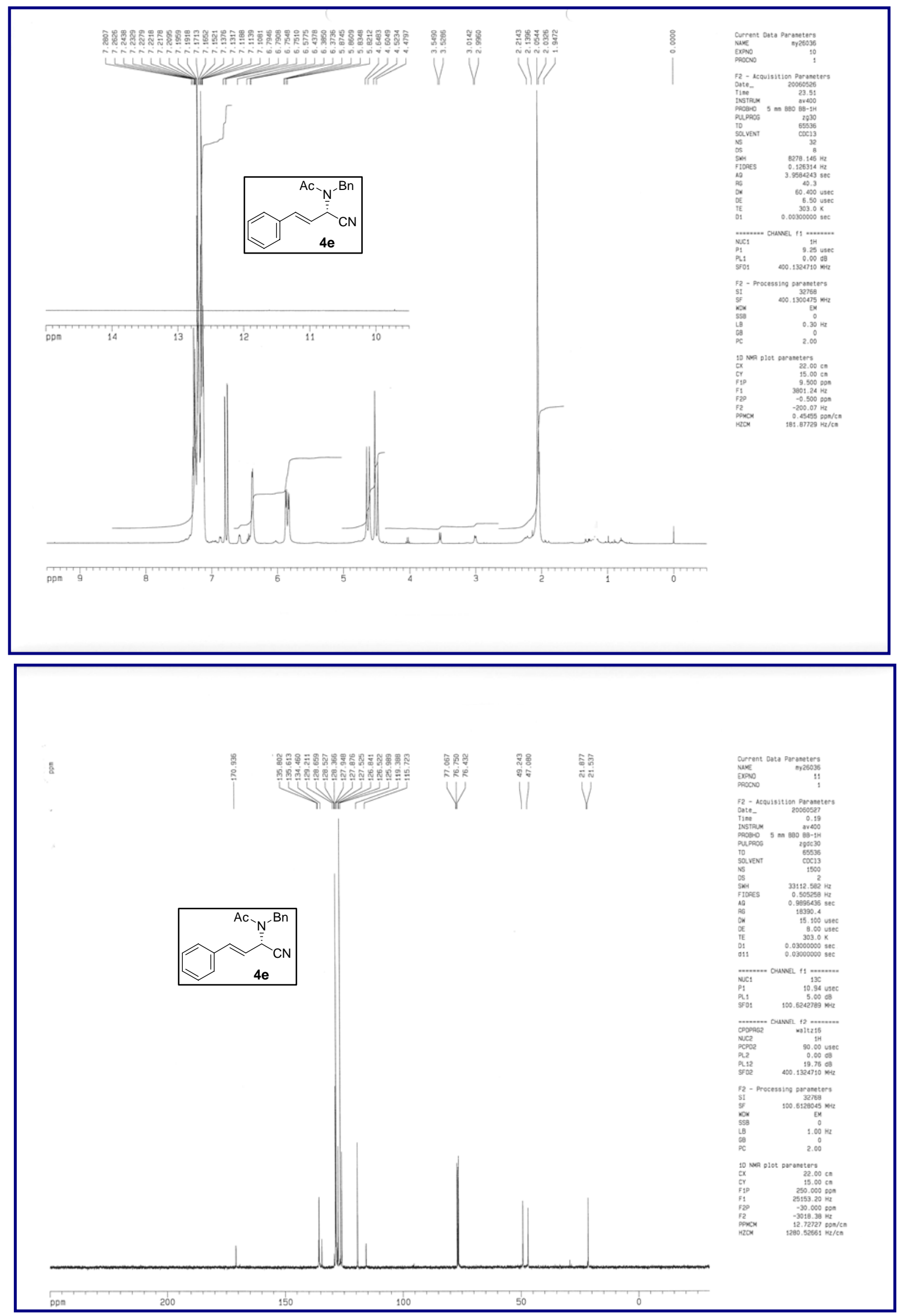

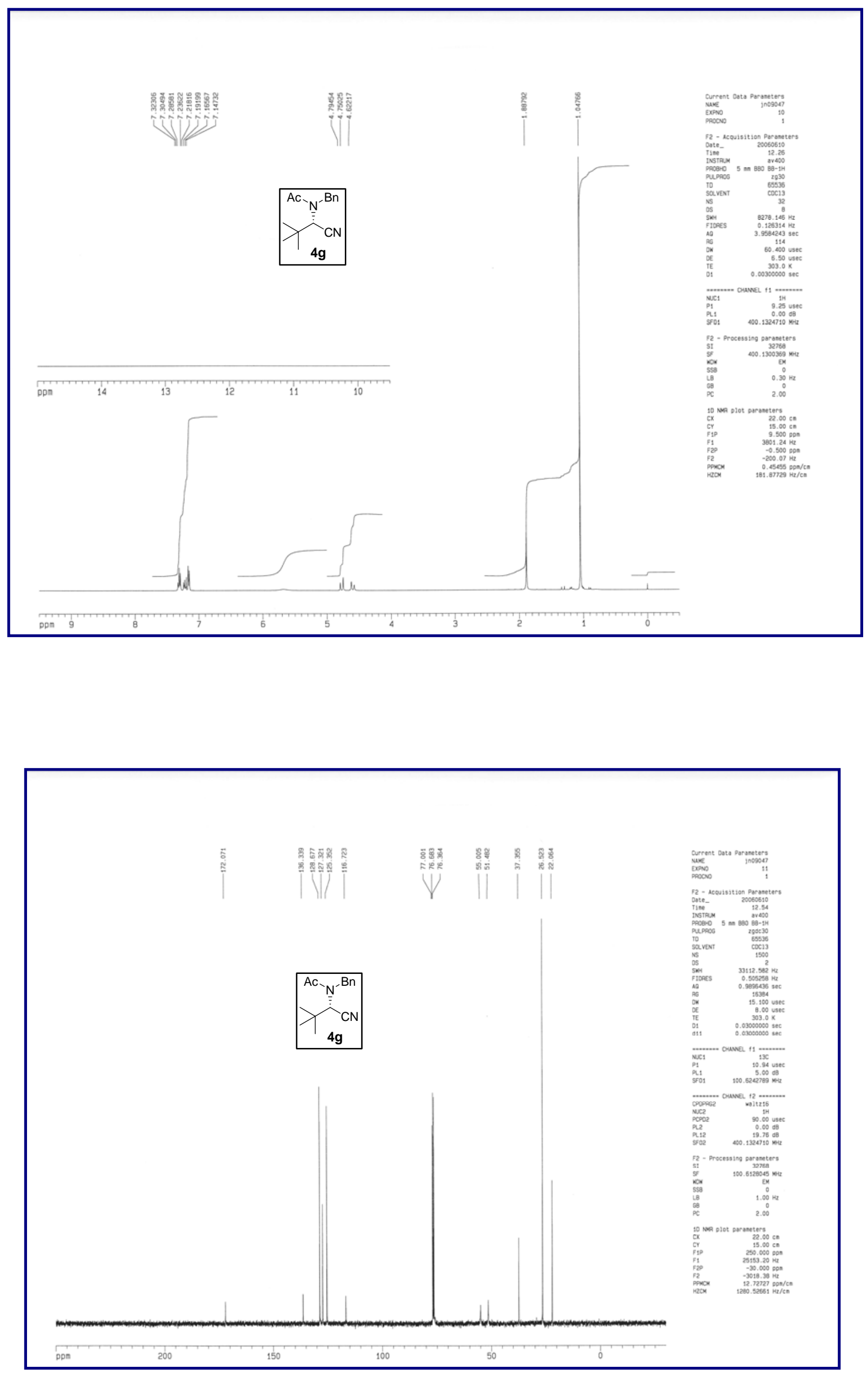

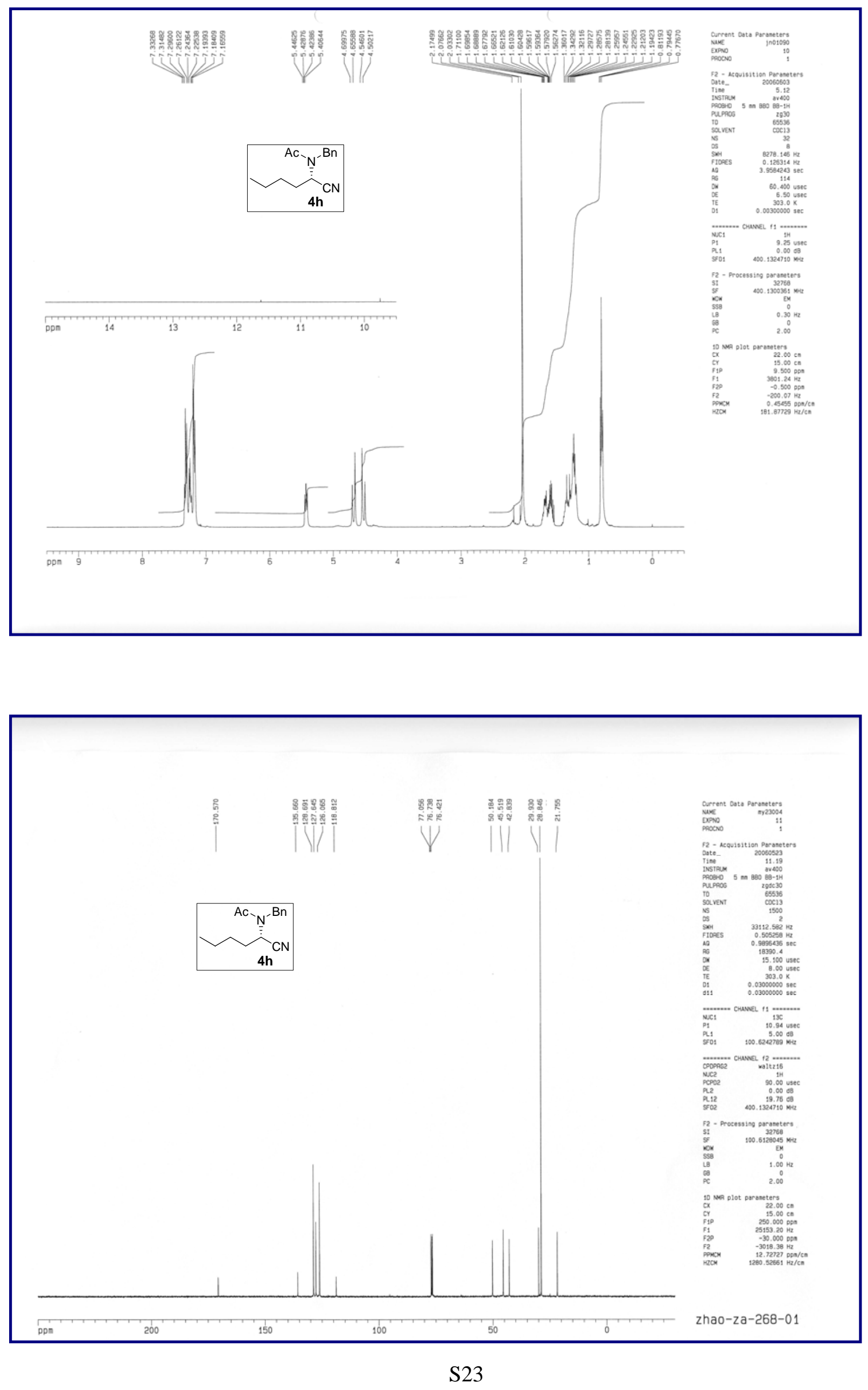


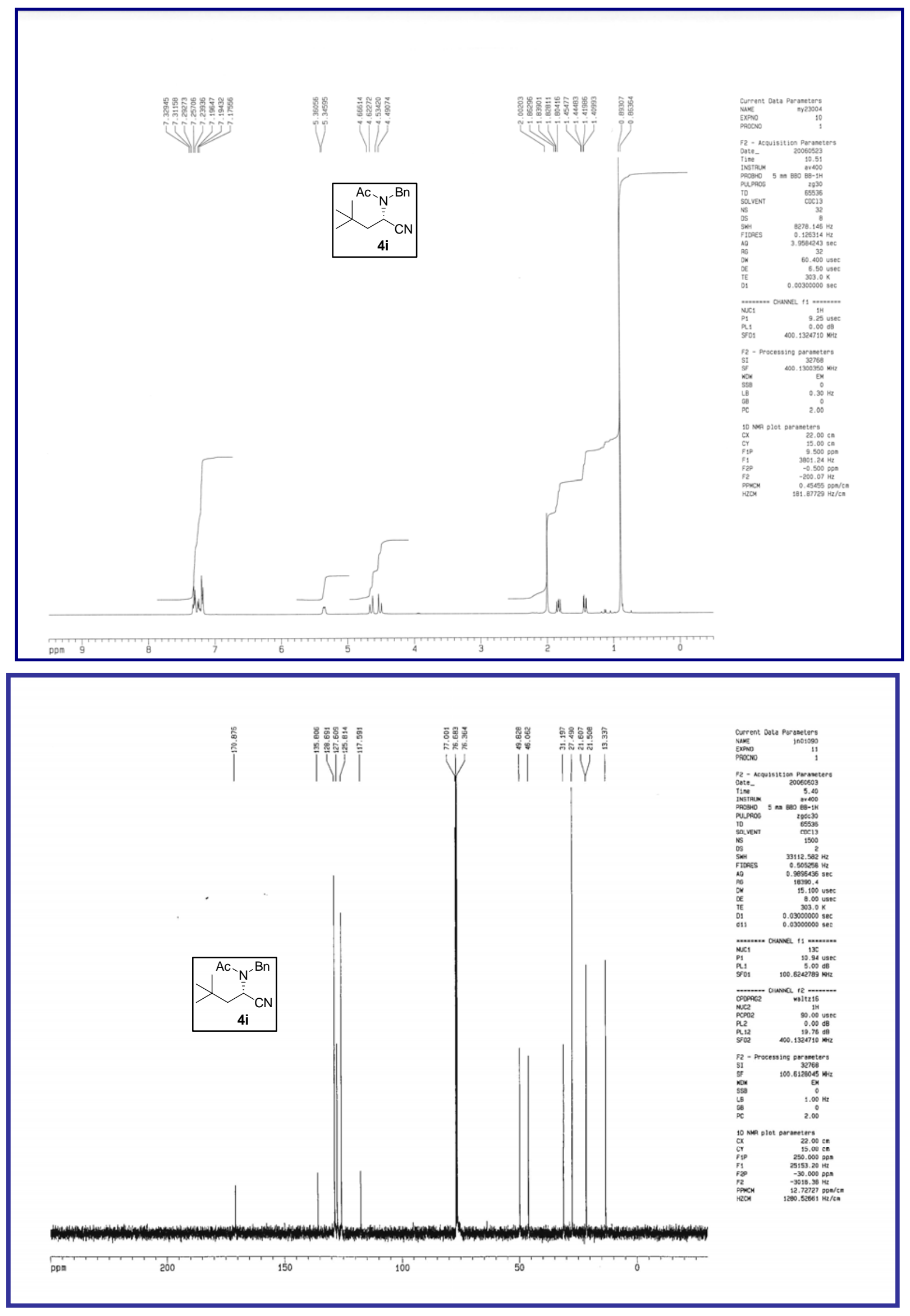



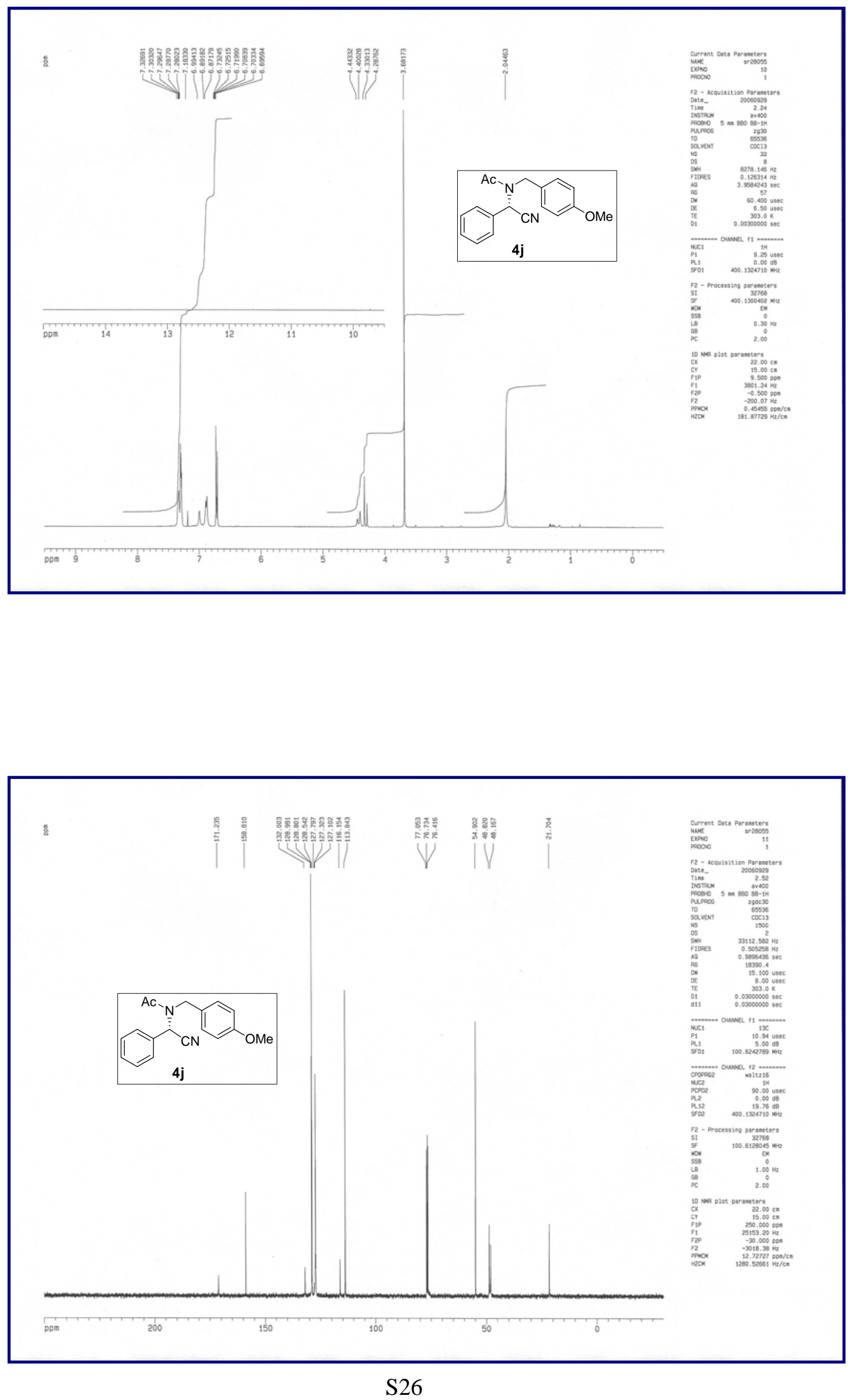

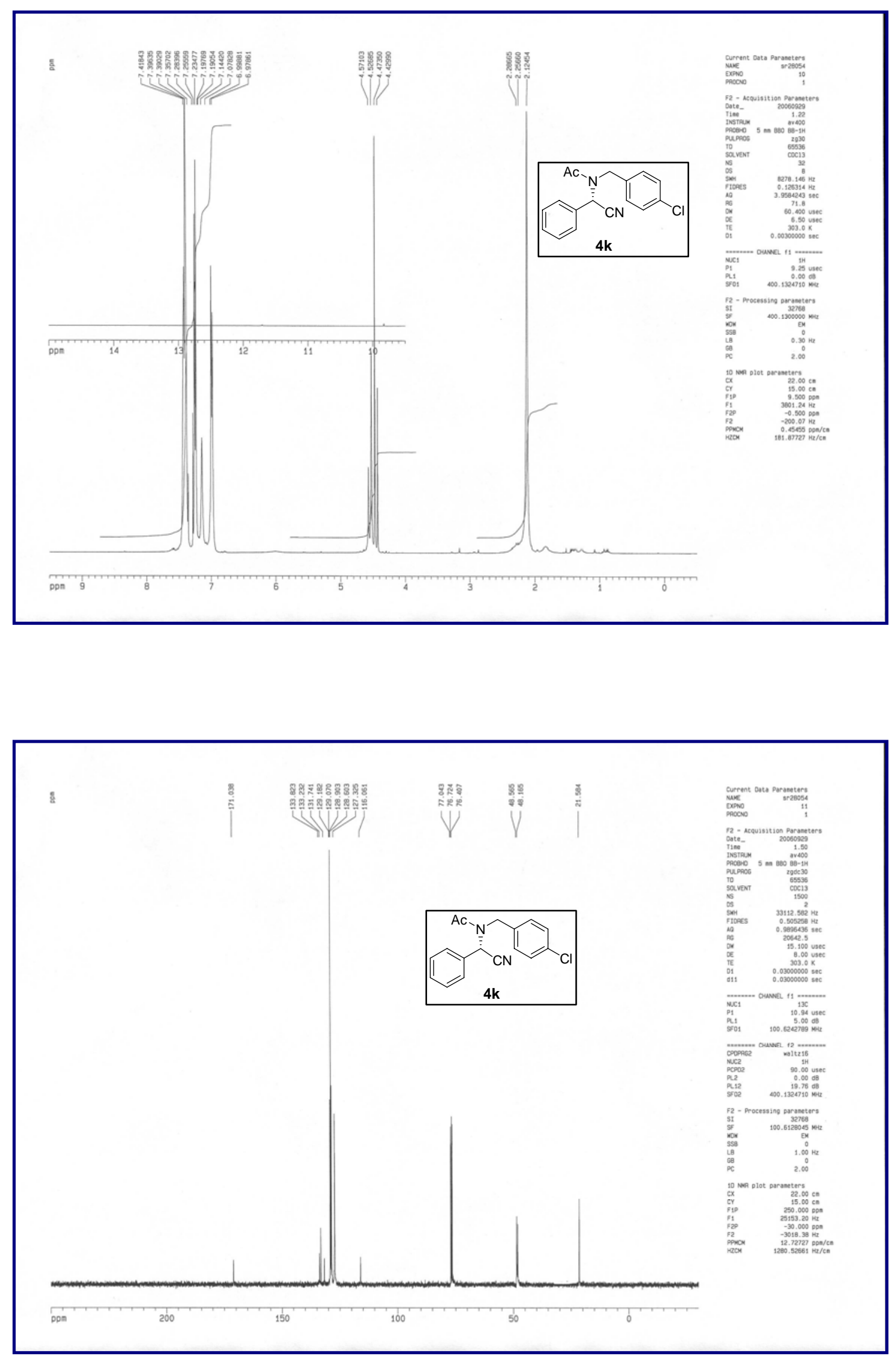

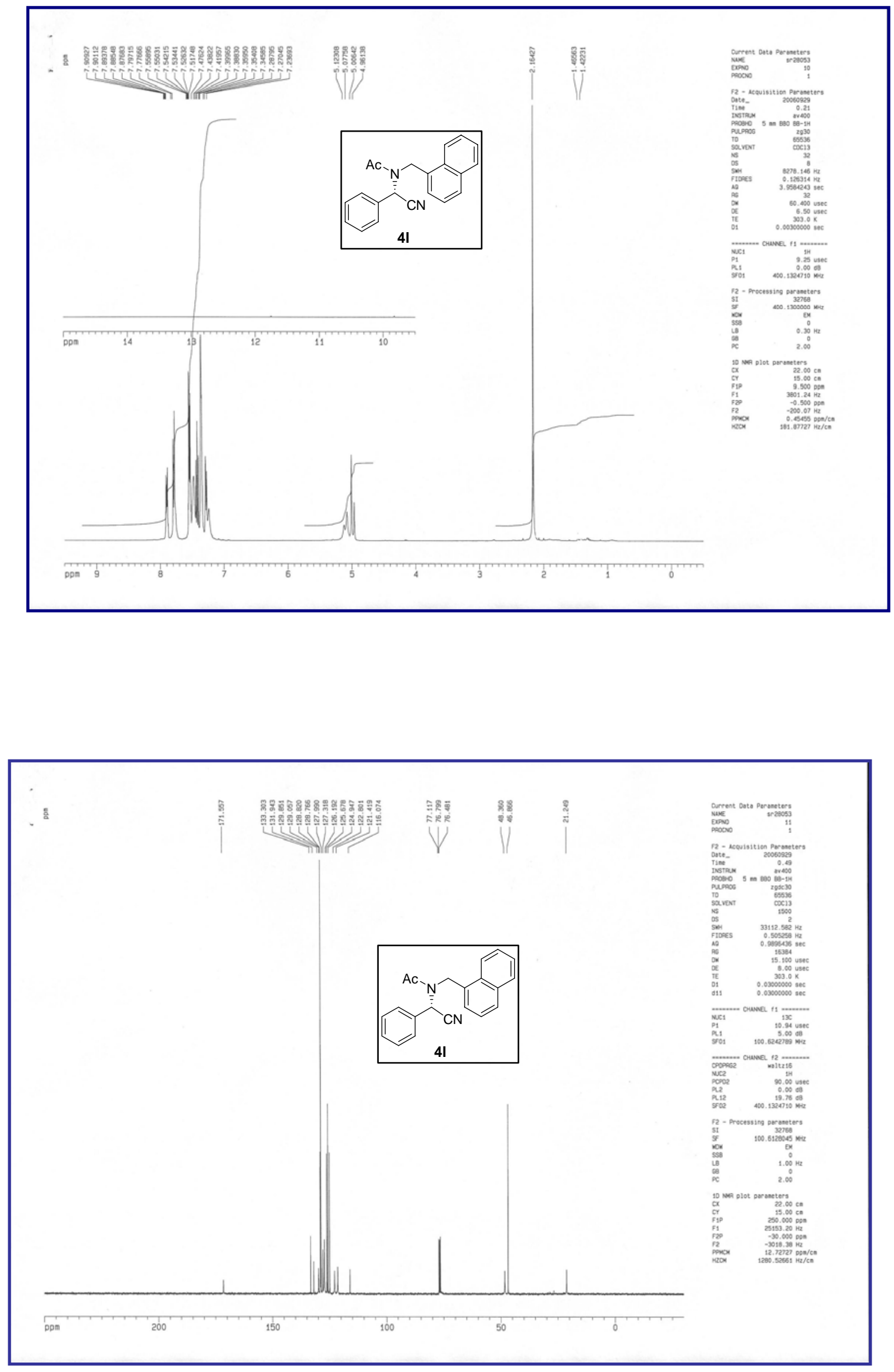

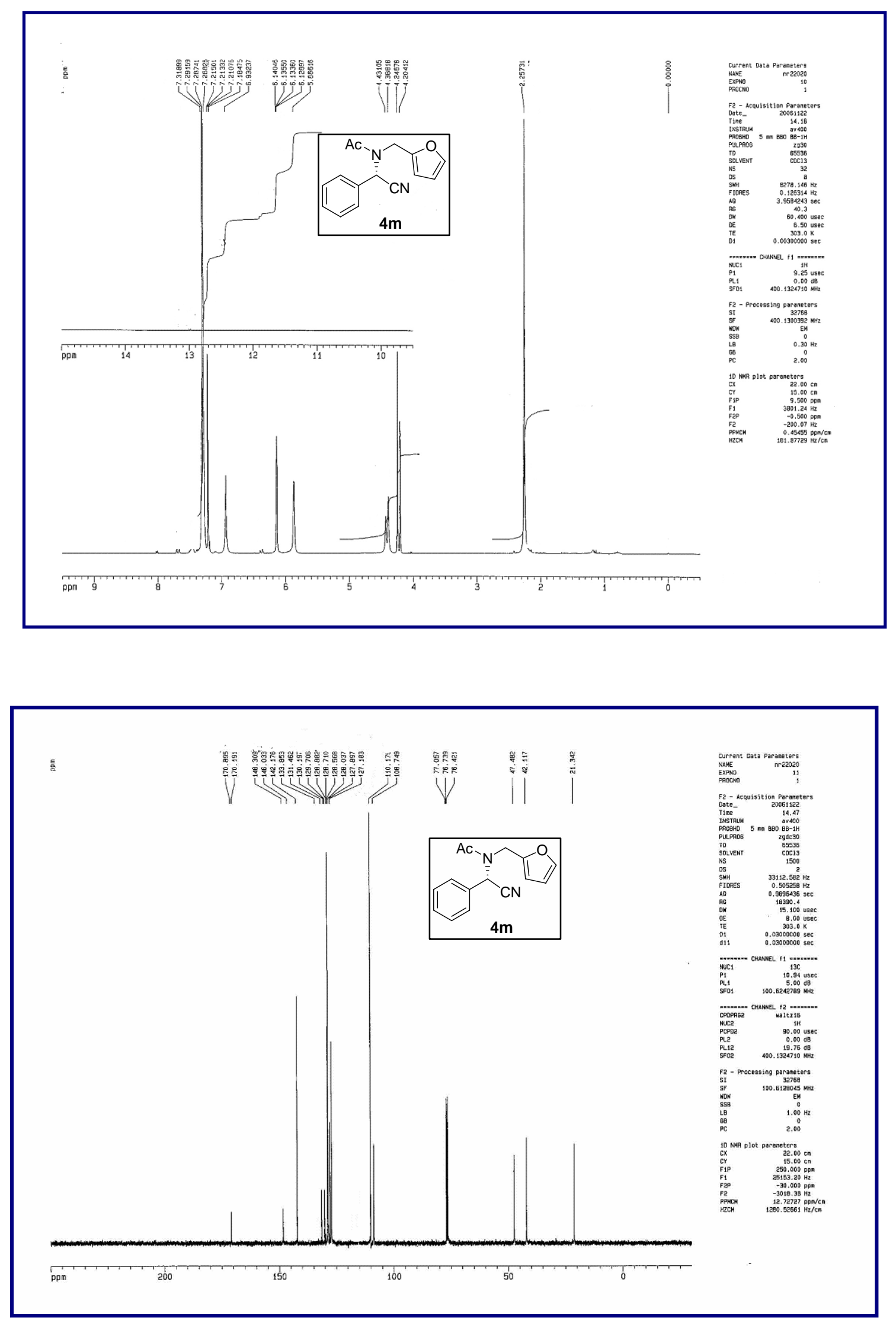

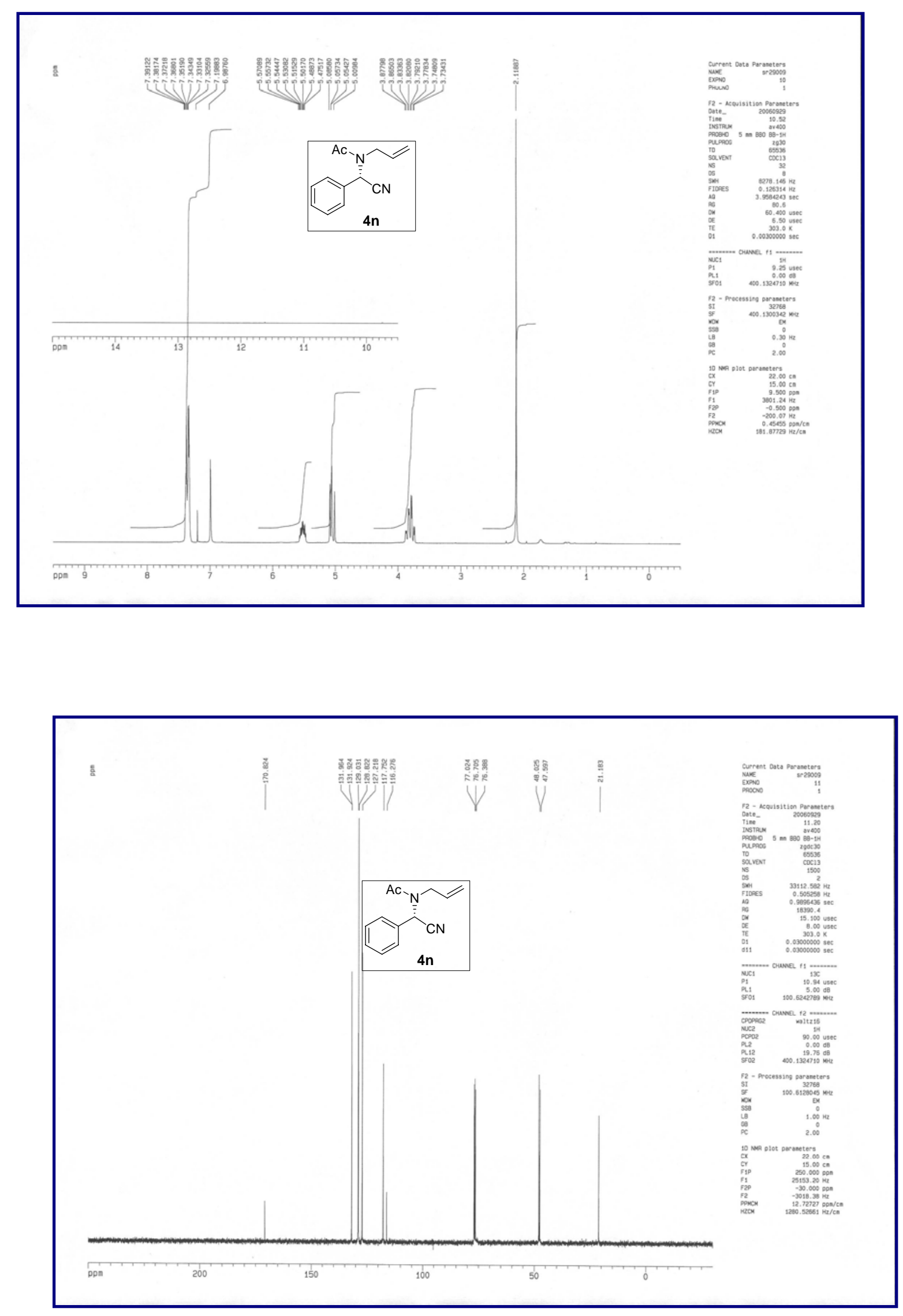

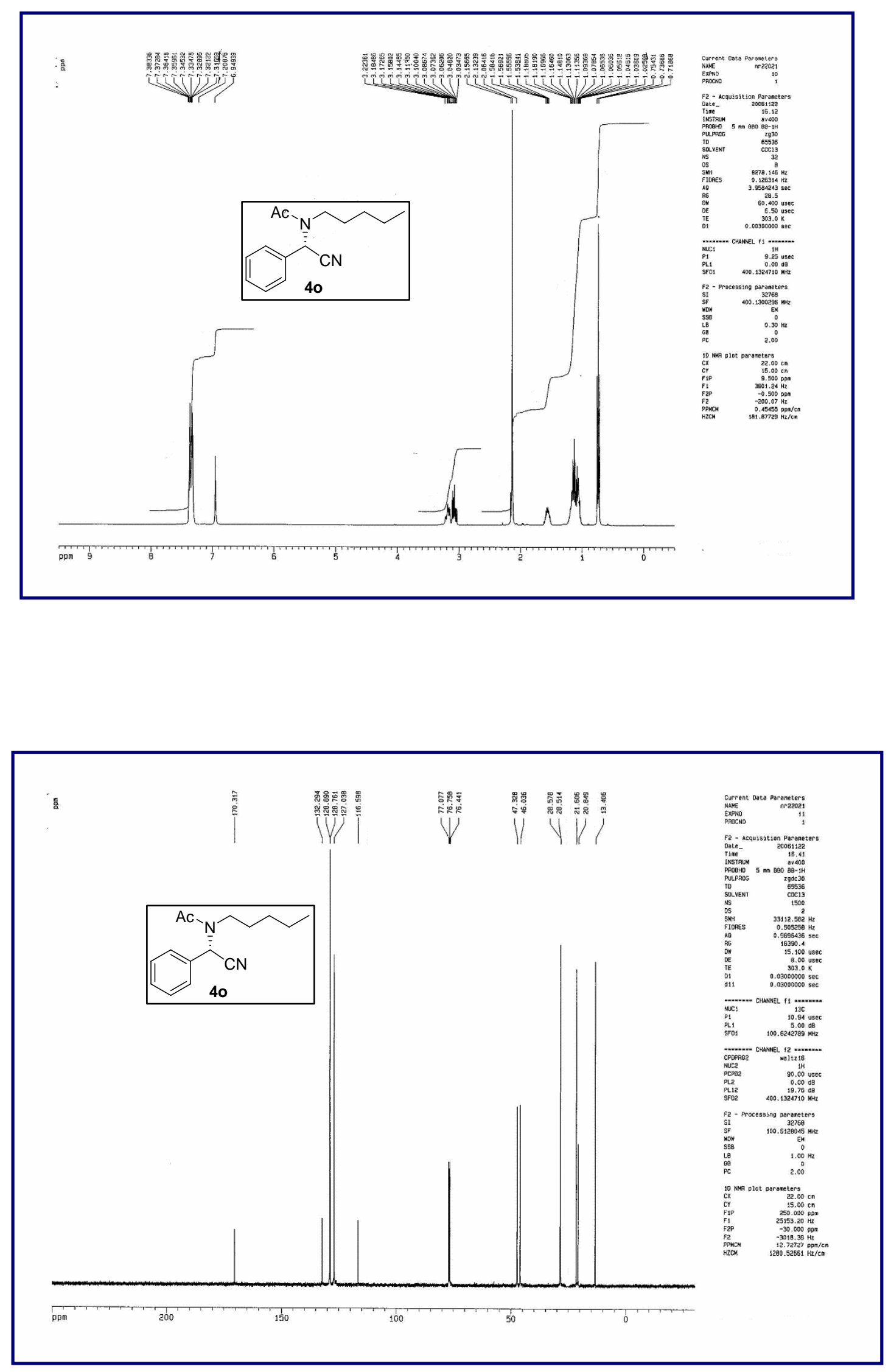


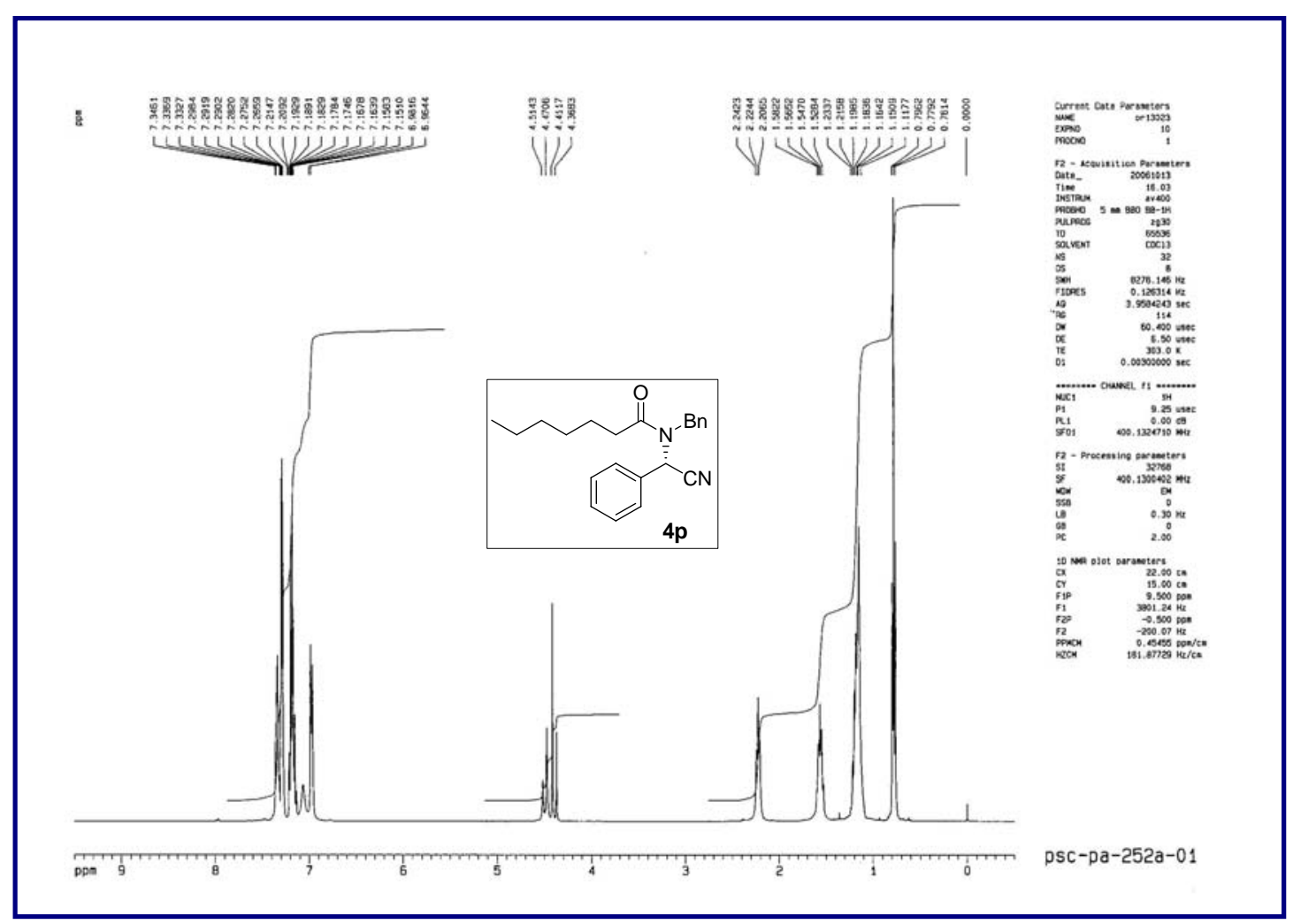

๕
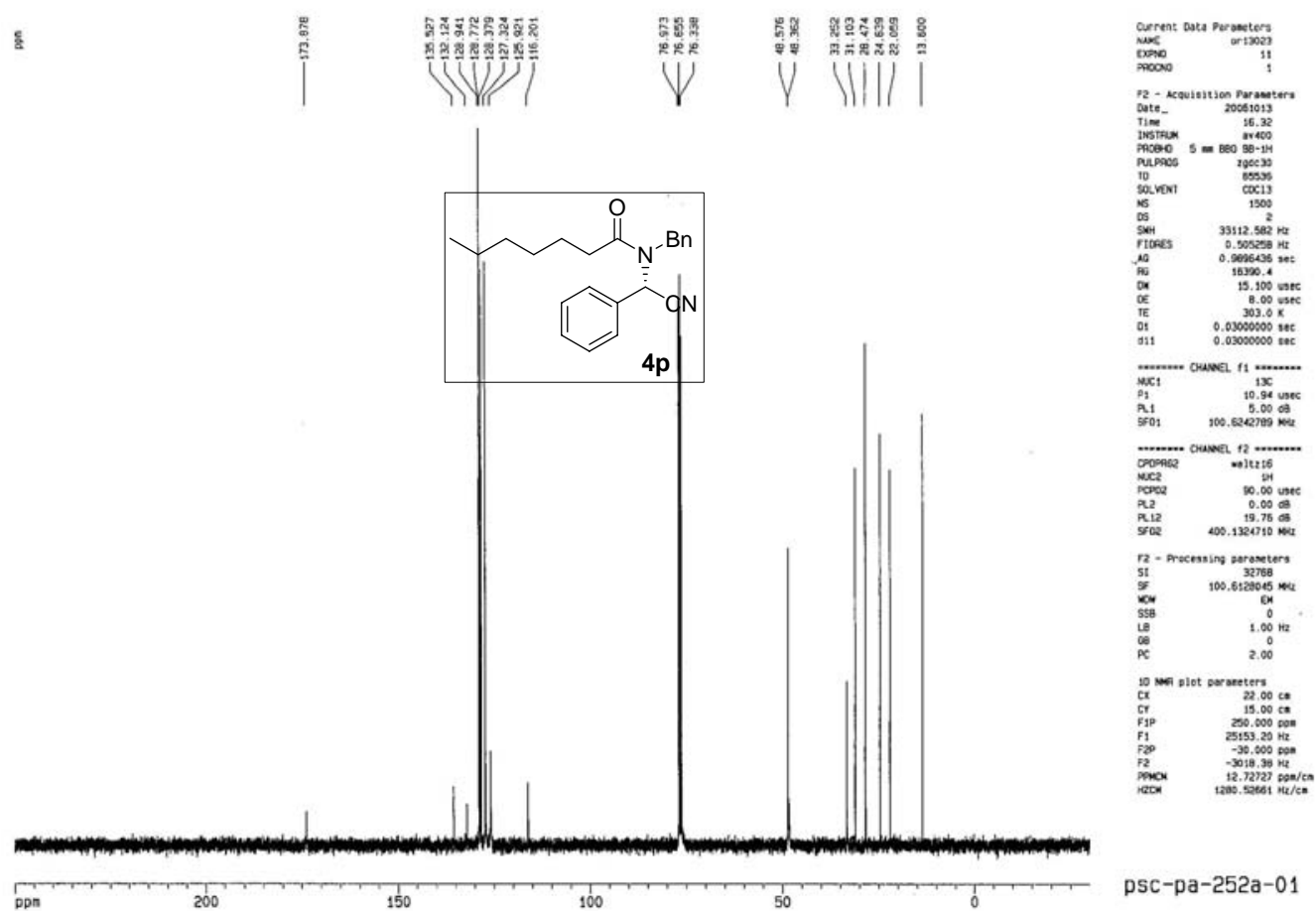